\title{
VINCULAÇÃO DE IMAGENS PARA BUSCA E VISUALIZAÇÃO A PARTIR DE SISTEMA DE INFORMAÇÃO EM RADIOLOGIA (RIS)
}

\author{
EDILSON CARLOS CARITÁ
}

Dissertação apresentada à Escola de Engenharia de São Carlos da Universidade de São Paulo, como parte dos requisitos para obtenção do Título de Mestre em Engenharia Elétrica.

Orientador: Prof. Dr. Paulo Mazzoncini de Azevedo Marques

São Carlos

2002 
A Deus pela vida e oportunidade de crescimento e realização deste trabalho.

Aos meus pais, pelo amor, carinho, companheirismo e apoio em todos os momentos de minha vida.

Ao meu irmão pelo apoio e amizade. 
Ao professor e amigo Paulo Mazzoncini de Azevedo Marques, meu orientador, pela orientação, apoio, cooperação e confiança oferecidos na realização deste trabalho.

À professora Annie France Frére Slaets, pelo apoio e colaboração em todos os momentos.

Ao ex-professor e amigo Márcio Alexandre Marques, que participou de minha formação acadêmica e incentivou para a realização deste trabalho.

Ao amigo Enzo Seraphim, que me incentivou para o ingresso no programa de pósgraduação.

Aos amigos do CHOPI: André, David, Lucas, Luciene, Marcelo Costa, Marcelo Honda e Roberto, pelo apoio e companheirismo.

Aos amigos do @ladim: Clayton, Flávio, Leandro, Marcelo, Silvia, Vivian e aos demais que fazem parte deste laboratório, pela amizade e presteza.

A todos os amigos e colegas conhecidos nesta etapa e aqueles que mesmo de longe torceram por mim.

Ao Departamento de Engenharia Elétrica da Escola de Engenharia de São Carlos da Universidade de São Paulo, pela oportunidade de realização deste trabalho.

Aos docentes e funcionários do Centro de Ciências das Imagens e Física Médica da Faculdade de Medicina de Ribeirão Preto da Universidade de São Paulo, pelo apoio e espaço para o desenvolvimento deste trabalho.

A Fundação de Amparo a Pesquisa do Estado de São Paulo (FAPESP), pelo apoio financeiro, processo $n^{\circ}$ 99/10875-5. 


\section{RESUMO}

Este trabalho apresenta o estudo e implementação de um Sistema para Vinculação e Visualização de Imagens de Ressonância Magnética Nuclear e Tomografia Computadorizada a partir de um Sistema de Informação em Radiologia (RIS), possibilitando a recuperação e disponibilização dos exames (laudos e imagens), através de rede "ethernet", para visualização a partir de uma interface desenvolvida para "browser".

Os exames podem ser recuperados através do número de registro (RGHC) ou do nome do paciente. As imagens utilizadas no trabalho estão nos padrões DICOM 3.0 ("Digital Imaging and Communications in Medicine") e JPEG ("Joint Photographic Experts Group”). Para a vinculação dos exames que possuem suas imagens em JPEG foi desenvolvida uma interface para inclusão das informações necessárias que garantem a consistência deste processo.

Para os exames que possuem suas imagens em formato DICOM 3.0 as informações foram extraídas automaticamente dos cabeçalhos e armazenadas no banco de dados.

O sistema possui uma interface amigável ao usuário, podendo ser rapidamente incorporada ao projeto de um PACS ("Picture Archiving and Communication System"). A implementação foi idealizada para servir ao Hospital das Clínicas da Faculdade de Medicina de Ribeirão Preto da Universidade de São Paulo (HCFMRP/USP), com base no seu Sistema de Informação em Radiologia.

Os resultados demonstram que o tempo de retorno das imagens é clinicamente satisfatório e considerado bom pela avaliação qualitativa dos médicos. 


\section{ABSTRACT}

This work presents the study and implementation of a system aiming the Indexing and Visualization of the Images of Nuclear Magnetic Resonance and Computerized Tomography from a Radiology Information System (RIS), allowing the retrieval and availability of the exams (results and images), through an ethernet net for visualization beginning with an interface developed to a browser.

The exams may be recovered either through their register number (RGHC) or the patient's name. The images employed in the work follow the DICOM 3.0 (Digital Imaging and Communications in Medicine) and JPEG (Joint Photographic Experts Group) patterns. For the indexing of the exams that present images in JPEG, an interface was developed to include the information required to guarantee the consistency of the process.

For the exams presenting the DICOM 3.0 format, information was extracted automatically from the heading and filed in the database.

The system has a friendly interface for the user, it may be rapidly incorporated to the project of an PACS (Picture Archiving and Communication System). The implementation was idealized to serve the Hospital das Clínicas da Faculdade de Medicina de Ribeirão Preto, of the Universidade de São Paulo (HCFMRP/USP), based on its Radiology Information System (RIS).

The results demonstrated that the time of retrieval of the images is satisfactory and considered good by evaluation qualified of the doctors. 


\section{SUMÁRIO}

Lista de Figuras................................................................................................................ iv

Lista de Tabelas................................................................................................................. vi

Capítulo 1 - Introdução...................................................................................................

1.1 Objetivos da Pesquisa..................................................................... 02

1.2 Organização da Dissertação................................................................. 03

Capítulo 2 - Radiologia sem filme (“Filmless")................................................... 05

Capítulo 3 - HIS - “Hospital Information System”..................................................

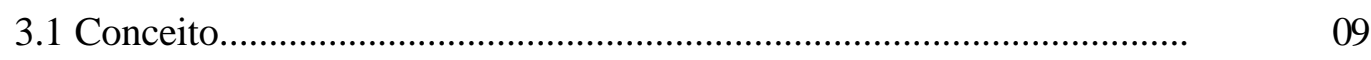

3.2 Benefícios de um Sistema de Informação Hospitalar.................................. $\quad 14$

Capítulo 4 - RIS - “Radiology Information System”.......................................... 16

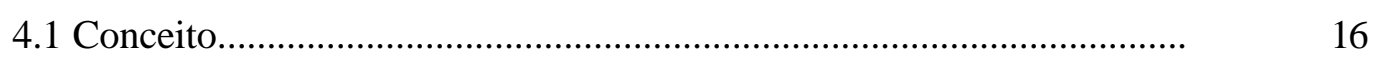

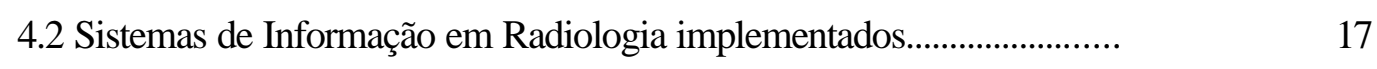

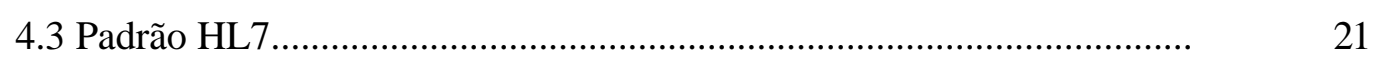

4.4 Sistema de Reconhecimento de Voz..................................................... 23

Capítulo 5 - PACS - “Picture Archiving and Communicatios System”.............. 24

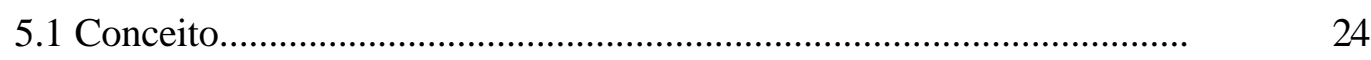

5.2 Substistemas de um PACS.............................................................. 26

5.3 Infra-Estrutura de um PACS.......................................................... $\quad 30$

5.4 O Pacs e a Web.............................................................................. 34

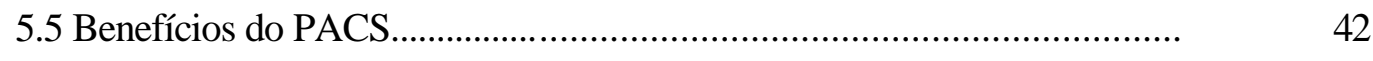




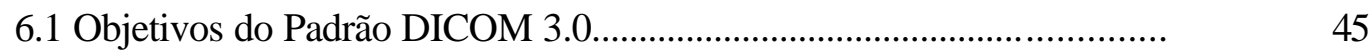

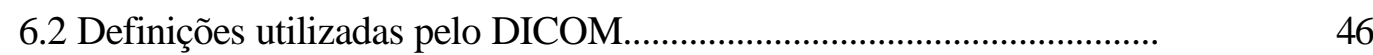

6.3 Serviços do padrão DICOM...................................................................

6.4 Suporte para rede DICOM............................................................ 54

6.5 Vantagens do padrão DICOM............................................................

Capítulo 7 - Banco de Dados................................................................................. $\quad 56$

7.1 Introdução................................................................................... 56

7.2 Modelo Entidade Relacionamento (ER).................................................. 59

7.3 Modelo Entidade Relacionamento Estendido (ERE).................................. 59

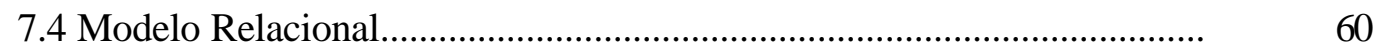

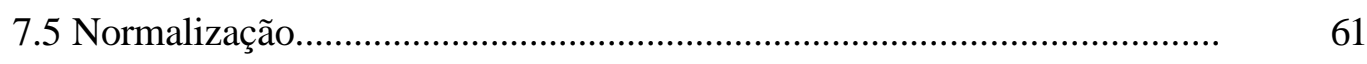

7.6 Álgebra Relacional.......................................................................... 61

Capítulo 8 - Materiais e Métodos............................................................................. 63

8.1 Sistema Operacional......................................................................... 63

8.2 Linguagens de Desenvolvimento........................................................... 64

8.3 Servidor "Web" "............................................................................ 65

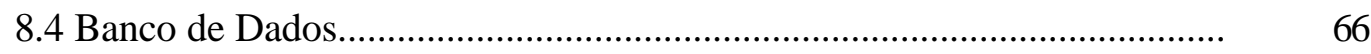

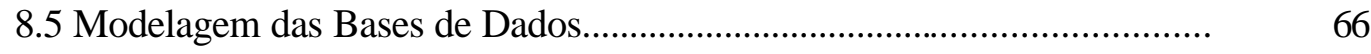

8.6 Interface para leitura e abertura dos arquivos DICOM.................................

8.7 Interface de Recuperação e Visualização...................................................... 73

8.8 “Applet Java” Visualizador DICOM...............................................................

Capítulo 9 - Resultados Obtidos e Discussão............................................................ 80

9.1 Recuperação das Imagens...................................................................... 80 
9.2 Avaliação pelos médicos especialistas...................................................... 85

9.3 Padronização na realização do exame........................................................... 87

Capítulo 10 - Conclusões.................................................................................. 89

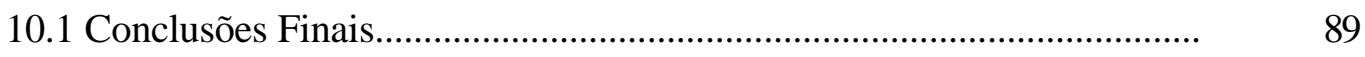

10.2 Sugestões para Trabalhos Futuros....................................................... $\quad 90$

Referências bibliográficas.................................................................................. 91 


\section{Lista de Figuras}

Figura 2.1 - Redução em número de estudos pendentes........................................... 06

Figura 2.2 - Redução em solicitação de novas imagens............................................ 07

Figura 3.1 - Computador centralizado com rede em estrela, em um hospital.......... 11

Figura 5.1 - Esquema estrutural do PACS ....................................................... 25

Figura 6.1 - Estruturas principais do modelo de informações do DICOM................

Figura 6.2 - Partes correntes do padrão DICOM e partes propostas para extensão do padrão............................................................................ 54

Figura 7.1 - Esquema básico da estrutura de um SBD........................................ 58

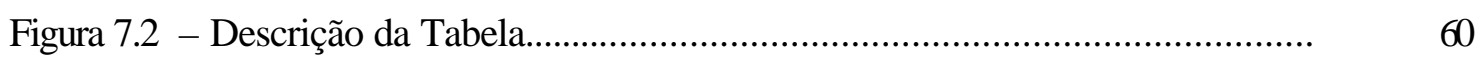

Figura 8.1 - Tela principal do Sistema do Livro Eletrônico........................................... 68

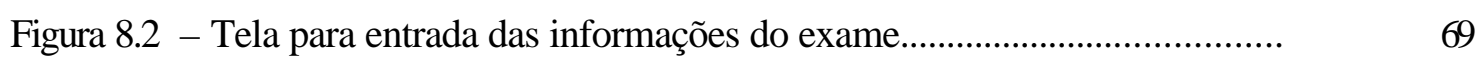

Figura 8.3 - Tela indicando que a inclusão foi realizada com sucesso......................... $\quad 70$

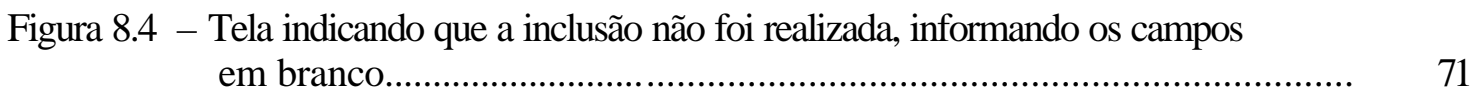

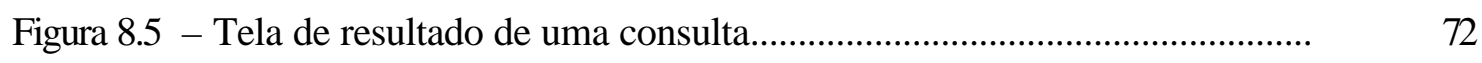

Figura 8.6 - Tela do Leitor das imagens do padrão DICOM....................................... 73

Figura 8.7 - Tela inicial do “Laudo Web” (Sistema de Vinculação e Visualização de Imagens Médicas)........................................................................ 74

Figura 8.8 - Resultado de uma consulta por Sobrenome de paciente........................... 75

Figura 8.9 - Resultado de uma consulta pelo número do Registro do paciente............. 75

Figura 8.10 - Resultado de uma consulta onde às imagens não foram encontradas........ 77

Figura 8.11 - Resultado de uma consulta onde às imagens foram retornadas................. 77

Figura 8.12 - Resultado da consulta aos laudos completos....................................... 78 
Figura 8.13 - Resultado da consulta as imagens onde o número do CD foi retornado... $\quad 78$

Figura 8.14 - Visualizador de Imagens DICOM..................................................... 79

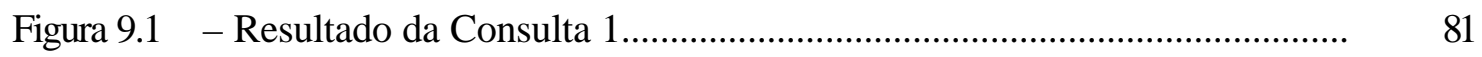

Figura 9.2 - Resultado da Consulta 2 .............................................................. 82

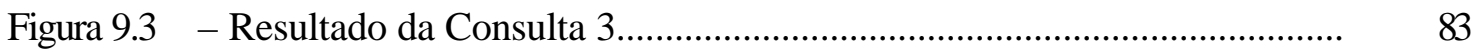

Figura 9.4 - Resultado da Consulta 4........................................................................ 84 


\section{Lista de tabelas}

Tabela 5.1 - Resultado da transferência para o novo RIS em dados estatísticos..... 32

Tabela 5.2 - Estimativa de custo por volume de armazenamento............................ 34

Tabela 6.1 - Exemplo de construção de uma Classe DICOM SOP........................... $\quad 50$

Tabela 6.2 - Resumo das partes principais do padrão DICOM 3.0......................... 53

Tabela 8.1 - Relação das tags utilizadas para o armazenamento no banco de dados $\quad 67$

Tabela 8.2 - Tabela Imagem, armazena as informações referentes à imagem........... $\quad 67$

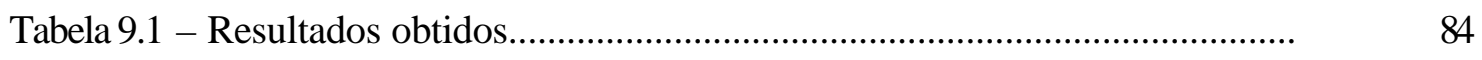

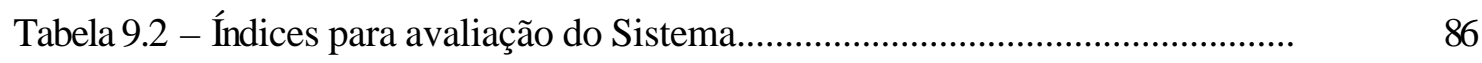

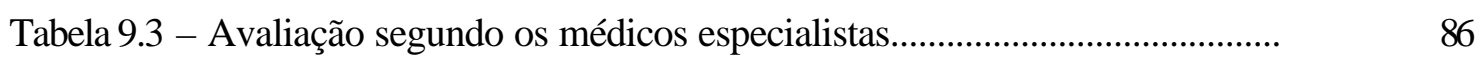

Tabela 9.4 - Resultado final da avaliação pelos médicos especialistas...................... $\quad 86$ 


\section{Capítulo 1}

\section{Introdução}

O avanço tecnológico tenta dia-a-dia aperfeiçoar e melhorar suas técnicas para auxiliar os médicos no diagnóstico por imagem. Com o advento das imagens digitais, hoje é possível realizar vários processamentos para mostrar de forma objetiva os pontos de interesse necessários para o diagnóstico médico. Porém, para que isso seja possível, essas imagens em formato digital e suas informações necessitam de um meio de gerenciamento e armazenamento.

Devido ao aumento do volume de dados gerados, tanto pelo número de exames realizados, quanto pela quantidade de modalidades disponíveis (Radiografia Digital, Tomografia Computadorizada, Ressonância Magnética, Medicina Nuclear e Ultra-som), o gerenciamento automatizado tornou-se praticamente uma necessidade básica.

Seguindo essa tendência, cada vez mais os hospitais de grande e médio porte estão integrando seus sistemas de informação. Para isso foram criados alguns conceitos e modelos adotados mundialmente, sendo estes: Sistema de Informação Hospitalar ("Hospital Information System - HIS"), Sistema de Informação em Radiologia ("Radiology Information System - RIS") e Sistema de Comunicação e Armazenamento de Imagens ("Picture Archiving and Communication System - PACS") [BAKKER-1991].

Para que esses sistemas pudessem ter êxito em seus propósitos foi definido, em 1993, um padrão para transferência de imagens e informações associadas, conhecido como DICOM 3.0 ("Digital Imaging and Communication in Medicine"). 
Atualmente o uso da WWW ("World Wide Web") tem crescido bastante na radiologia, seja para ensino, pesquisa ou assistência. Essa tecnologia está sendo usada principalmente para acessar informações e imagens integradas em redes PACS e para teleradiologia [FERNÀNDEZ-BAYÓ-2000].

A utilização de todo esse aparato tecnológico possibilitou o surgimento do conceito de Radiologia "Filmless" nos hospitais. Radiologia "Filmless" consiste em um sistema onde o filme radiológico foi eliminado quase que por completo dos serviços de radiodiagnóstico.

A Radiologia "Filmless" traz melhoria no que diz respeito à acessibilidade na aquisição, exibição e processamento de imagens e pode reduzir custos, pois não consome filmes e praticamente elimina a necessidade de repetição de exames, devido a erros de técnicas ou extravio. Porém é preciso estruturar uma forma de armazenamento e distribuição de informação consistente e segura.

\subsection{Objetivos da Pesquisa}

O objetivo principal deste trabalho foi o estudo e desenvolvimento de um sistema para vinculação, recuperação e disponibilização de imagens médicas, para visualização em computadores ligados a rede "ethernet". A proposta foi vincular as informações dos pacientes com o laudo dos exames e suas respectivas imagens, para estudo e pesquisa de casos já laudados, bem como para consulta e revisão de exames.

Outra proposta desta pesquisa era estudar o protocolo DICOM, seu funcionamento e suas características, bem como adquirir conhecimentos para implementação de um servidor 
de imagens DICOM e tecnologias necessárias para a estruturação de um mini-PACS junto ao Serviço de Radiodiagnóstico do Hospital das Clínicas da Faculdade de Medicina de Ribeirão Preto, visando futuramente à possibilidade da implementação de um sistema de Radiologia "Filmless".

\subsection{Organização da Dissertação}

Esta dissertação está dividida em 10 (dez) capítulos. O primeiro capítulo traz uma introdução e apresenta os objetivos e organização desta pesquisa.

O segundo capítulo apresenta uma revisão de trabalhos considerando o conceito Radiologia sem filme (“Filmless”).

O terceiro capítulo apresenta uma revisão bibliográfica de trabalhos sobre o conceito de HIS - “Hospital Information System”.

O quarto capítulo apresenta uma revisão bibliográfica de trabalhos sobre o conceito de RIS - “Radiology Information System”.

O quinto capítulo apresenta a revisão bibliográfica de trabalhos e implementações de sistemas PACS - "Picture Archiving and Communication System".

O sexto capítulo apresenta a revisão bibliografia de trabalhos e considerações sobre o padrão DICOM - "Digital Imaging and Communication in Medicine”.

No sétimo capítulo é apresentada uma introdução sobre Banco de Dados e os modelos utilizados para sua implementação.

O oitavo capítulo descreve os materiais e métodos utilizados na implementação deste trabalho. 
O nono capítulo apresenta os resultados e discussão deste trabalho.

No décimo capítulo encontram-se as conclusões e também sugestões para trabalhos futuros sobre o assunto.

Ao final são apresentadas as referências bibliográficas estudadas. 


\section{Capítulo 8}

\section{Materiais e Métodos}

Neste capítulo são descritos os principais métodos e equipamentos empregados na realização deste trabalho de mestrado.

\subsection{Sistema Operacional}

A revisão bibliográfica mostra que a grande maioria dos sistemas HIS, RIS e PACS foram ou estão sendo implementados utilizando o ambiente Operacional "Windows $N T^{\prime \prime}$. Em nosso caso, devido ao Sistema de Informação em Radiologia (Laudo Eletrônico) do Hospital das Clínicas de Ribeirão Preto utilizar a plataforma "Windows", optamos pelo Sistema Operacional "Windows 2000 Professional".

O "Windows 2000 Professional" é um sistema operacional baseado no sistema

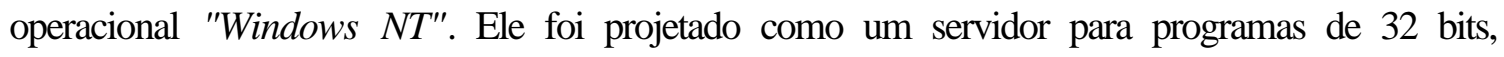
oferecendo diversos recursos de rede, sendo os mais importantes o DCOM ("Distributed Common Object Model”) e o DNS (“Domain Name Service”).

O "Windous 2000" funciona em redes heterogêneas utilizando uma combinação de protocolos TCP/IP, IPX/SPX e até NetBEUI, podendo com isso executar vários protocolos de rede simultaneamente. O "Windows 2000" está sendo muito usado como sistema operacional de servidores "Web", devido ao baixo custo de hardware e software necessários 
para configurar um site $^{3}$. Outros benefícios também bastante explorados são os recursos de “intranets”, devido à proliferação das redes WAN (“Wide Area Network”).

O "Windows 2000" aprimorou muitas características para seus usuários. Essas características incluem multi-tarefas, suporte de memória, multiprocessador simétrico, "plug and play”, “clustering”, NTFS (“NT File System”), serviço de qualidade, serviços terminais e serviços de instalação remoto.

\subsection{Linguagens de Desenvolvimento}

No desenvolvimento do algoritmo para abertura, leitura e transferência das informações do padrão DICOM para o banco de dados, partimos da necessidade de uma linguagem que fosse robusta e genérica.

A linguagem escolhida foi o $\mathrm{C}+$ "Builder", uma linguagem orientada a objetos com interface gráfica e conexão a banco de dados.

Sistemas disponibilizados em "browser" são uma estratégia mundial de disponibilização de informações de maneira segura, prática e rápida, se comparada com os métodos tradicionais. Outra vantagem proporcionada pelo "browser" é que o usuário não precisa ter o sistema instalado na sua máquina, basta apenas ter o "browser" e acesso ao sistema de consulta.

Visando esta tecnologia escolhemos a linguagem PHP ("Personal Home Page"), para implementação do sistema. PHP é uma linguagem que permite criar páginas "Web" dinâmicas, aumentando a interação com o usuário através de formulários, parâmetros da URL ("Uniform Resource Locator") e "links". Desta maneira é possível interagir com

\footnotetext{
${ }^{3}$ Site - Palavra em inglês que significa local, lugar. Na internet designa um conjunto de páginas que representa uma pessoa, instituição ou empresa na rede. O termo home page é usado para a página principal de um site.
} 
banco de dados e aplicações existentes no servidor, com a vantagem de não expor o código fonte para o cliente. Isso é útil quando o sistema está lidando com senhas ou qualquer tipo de informação confidencial.

O PHP é escrito na linguagem C ou Perl e o código fonte fica embutido no próprio HTML (“Hyper Text Markup Language”).

O PHP é uma das novas tecnologias de acesso a banco de dados via “Web”, ele vem do mesmo conceito que o CGI ("Commom Gateway Interface") e ASP ("Active Server Pages").

O PHP tem como uma das características mais importantes o suporte a um grande número de bancos de dados, como dBase, Interbase, mSQL, mySQL, Oracle, Sybase, PostgreSQL e vários outros. Também tem suporte a outros serviços através de protocdos como IMAP, SNMP, NNTP, POP3 e HTTP e possibilita abrir "sockets" e interagir com outros protocolos.

\subsection{Servidor "Web"}

Para o gerenciamento das páginas dinâmicas utilizamos como servidor HTTP 4 ("Hyper Text Transfer Protocol”), o software "Apache HITP Server versão 1.3.14", que é um servidor "Web" de código aberto, disponibilizado pela "Apache Software Foundation". Ele é gratuito e roda em diferentes plataformas, é atualmente um dos servidores "web" mais usado. Por possuir seu código aberto, facilita algumas alterações para melhorar seu desempenho, além de ser considerado estável, poderoso e flexível.

\footnotetext{
${ }^{4}$ Protocolo utilizado pelos computadores ligados à "Web" para comunicar-se entre si.
} 


\subsection{Banco de Dados}

Neste projeto utilizamos o Sistema de Gerenciamento de Banco de Dados "Oracle Enterprise Edition Release 8.1.6.1.0", produto da "Oracle Corporation?" para plataforma Intel com o sistema Operacional GNU/Linux como servidor. $\mathrm{Na}$ estação cliente, que utiliza o Sistema Operacional "Windows 2000", o software "Oracle Enterprise Manager Release 2.1.0.1.0”, auxilia no gerenciamento das informações. O Gerenciador de Banco de Dados está instalado em um IBM Server xSeries 230 (Pentium III) $900 \mathrm{MHz}, 1 \mathrm{~GB}$ RAM e 218.4GB de espaço em disco.

Uma parceria com o Centro de Informações e Análise do Hospital das Clínicas da FMRP possibilitou a importação da estrutura do banco de dados do Hospital, juntamente com suas informações, criando um ambiente de testes que não interfira em absolutamente nada no andamento normal dos serviços e possibilite uma futura integração das pesquisas realizadas no CCIFM (Centro de Ciências das Imagens e Física Médica) com a rotina hospitalar, como proposto por [AZEVEDO-MARQUES-2000].

\subsection{Modelagem das Bases de Dados}

Na modelagem da Base de Dados para o gerenciamento das imagens, consideramos as informações necessárias na vinculação dos exames com suas imagens e as informações contidas nos arquivos do padrão DICOM, já que esses arquivos contêm informações do exame tais como: identificador único, data da realização do exame, nome do paciente, 
idade, entre outras. Baseado nas informações descritas acima, criamos a tabela "IMAGEM", que é formada pelos atributos descritos na tabela 8.1.

\begin{tabular}{|l|l|c|}
\hline Nome do Campo & Tipo do campo & Tamanho do Campo \\
\hline NUM_EXAME & Numérico & 06 \\
\hline DIG_EXAME & Numérico & 03 \\
\hline COD_PACIENTE & Carácter & 08 \\
\hline DATA_ESTUDO & Data & 08 \\
\hline MODALIDADE & Caracter & 02 \\
\hline NUM_SERIE & Numérico & 03 \\
\hline NUM_IMAGEM & Numérico & 03 \\
\hline NUM_AQUISIÇÃO & Numérico & 03 \\
\hline ALTURA & Numérico & 03 \\
\hline LARGURA & Numérico & 03 \\
\hline IMAGEM & Caracter & 55 \\
\hline DICOM & Caracter & 01 \\
\hline CENTER & Numérico & 03 \\
\hline WIDTH & Numérico & 03 \\
\hline
\end{tabular}

Tabela 8.1 - Tabela Imagem, armazena as informações referentes a imagem.

Para controlar o acesso ao sistema criamos uma tabela que armazena as informações dos usuários do sistema, sendo os atributos dessa tabela apresentados na tabela 8.2.

\begin{tabular}{|l|l|c|}
\hline Nome do Campo & Tipo do campo & Tamanho do Campo \\
\hline LOGIN & Caracter & 10 \\
\hline SENHA & Caracter & 08 \\
\hline NOM_MEDICO & Caracter & 50 \\
\hline SBN_MEDICO & Caracter & 30 \\
\hline DATA_NASCIMENTO & Date & 08 \\
\hline NUM_CRM & Numérico & 06 \\
\hline
\end{tabular}

Tabela 8.2 - Tabela Usuário, armazena as informações referentes aos usuários do sistema.

O objetivo principal do trabalho foi desenvolver métodos para consultar os exames e suas imagens que estão armazenadas na Base de Dados, e que eventualmente possam ser disponibilizadas para visualização “on-line”. Porém, os exames de Ressonância Magnética 
recebem um número interno que é utilizado pra referenciar em qual $\mathrm{CD}$ estão armazenadas as imagens do exame, essas informações são transcritas em livros. Para melhorar a performance do sistema de vinculação e visualização das imagens, implementamos um sistema para inserção e consulta de forma informatizada desse livro, o qual é acessado pela internet pelos usuários autorizados (Figura 8.1).

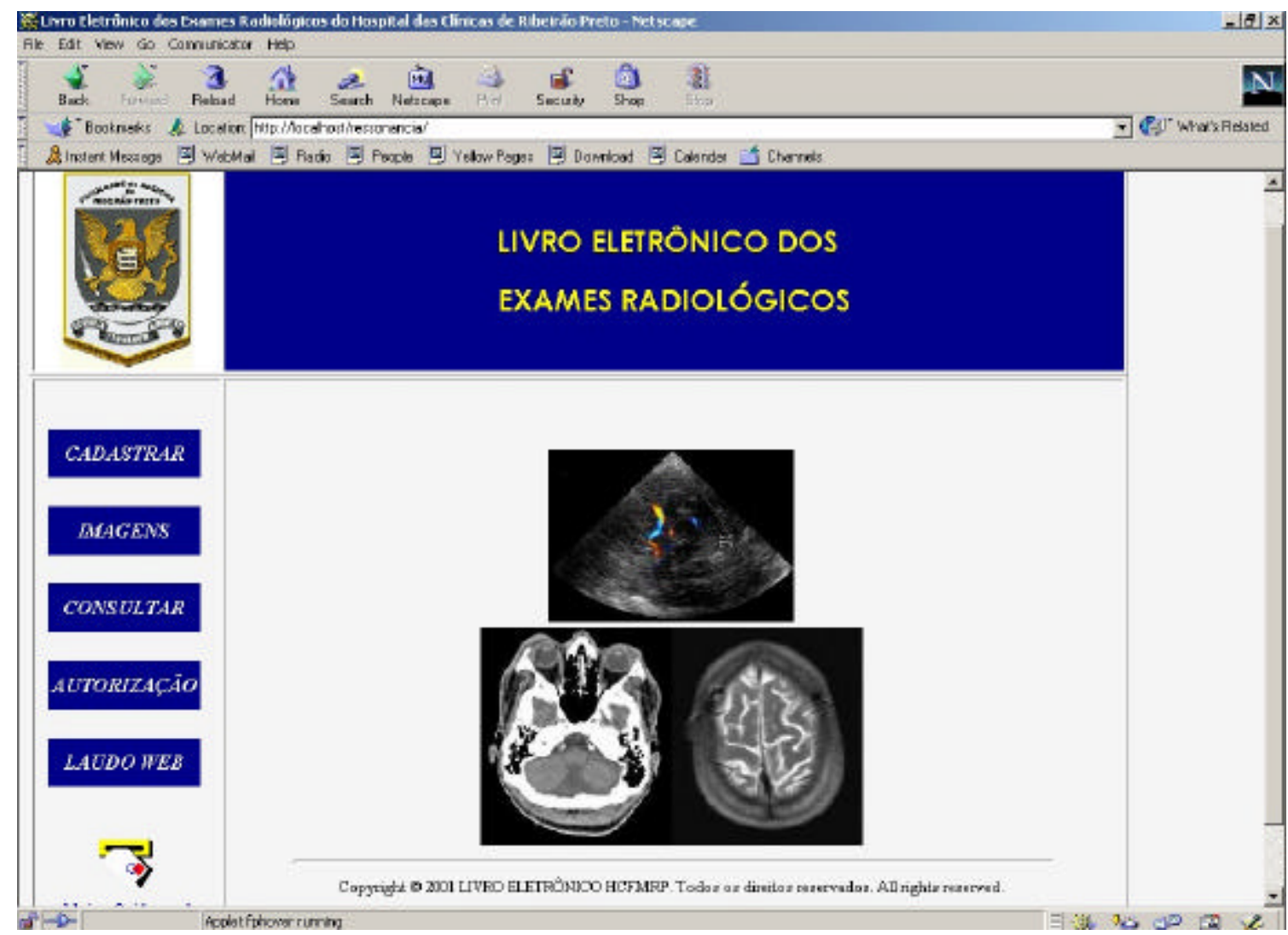

Figura 8.1 - Tela principal do Sistema do Livro Eletrônico.

O sistema foi desenvolvido para rodar em "browser", utilizando HTML, PHP e o gerenciador de Banco de Dados "ORACLE". O sistema possui uma tela principal que permite aos usuários o acesso para incluir as informações do livro da ressonância, consultalas, pedir autorização para utilizar o Livro Eletrônico, incluir as informações necessárias 
para a vinculação das imagens não DICOM (Tomografia Computadorizada) e um "link" para acessar o sistema de visualização das imagens.

Além dos exames de Ressonância Magnética, futuramente os exames de outras modalidades poderão utilizar esse sistema para controlar o armazenamento das imagens em $\mathrm{CD}$, até que se adote uma estrutura adequada, como um PACS.

As figuras 8.2 e 8.3 apresentam as telas para inclusão dos exames no Livro Eletrônico.

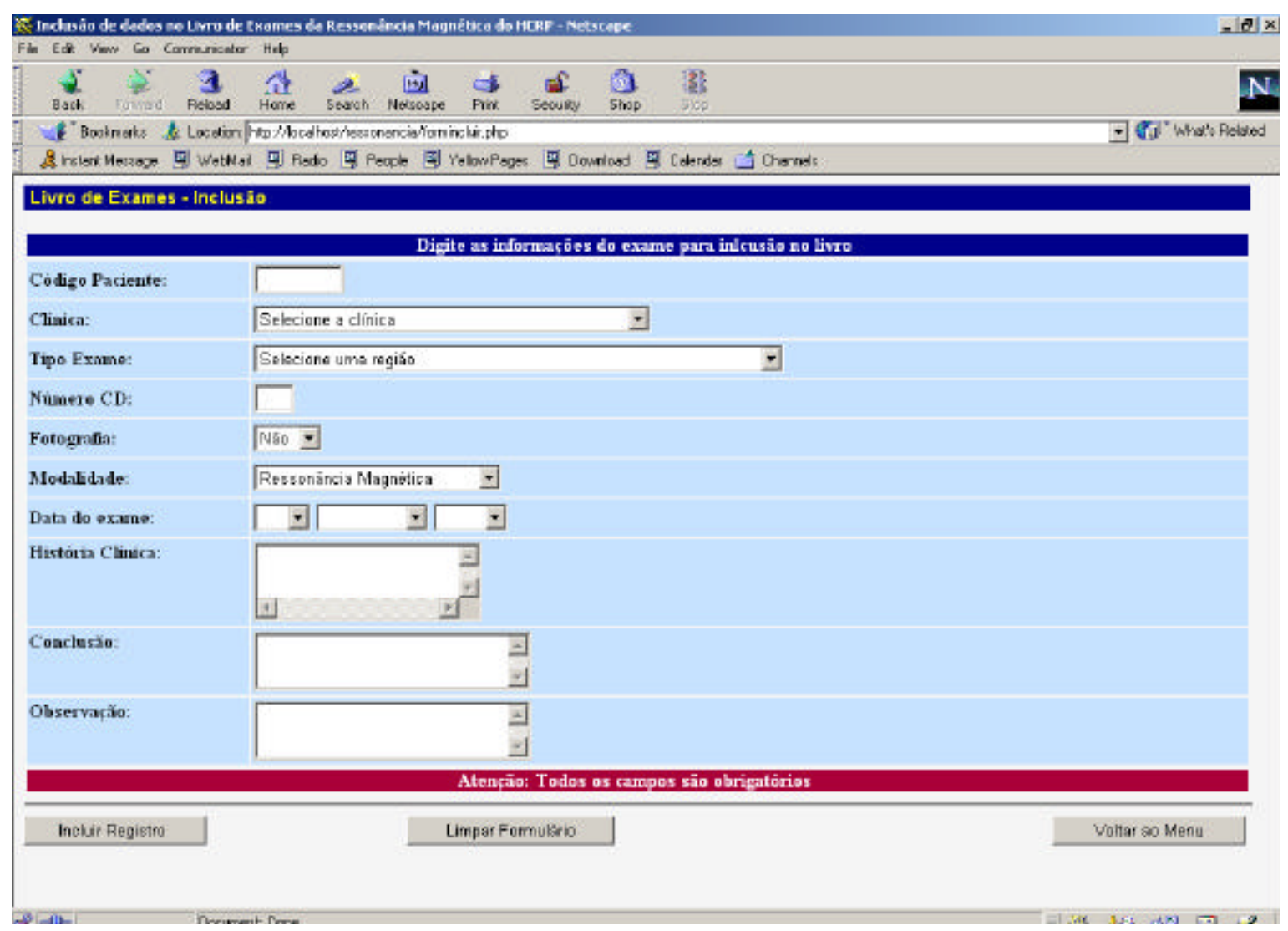

Figura 8.2 - Tela para entrada das informações do exame. 


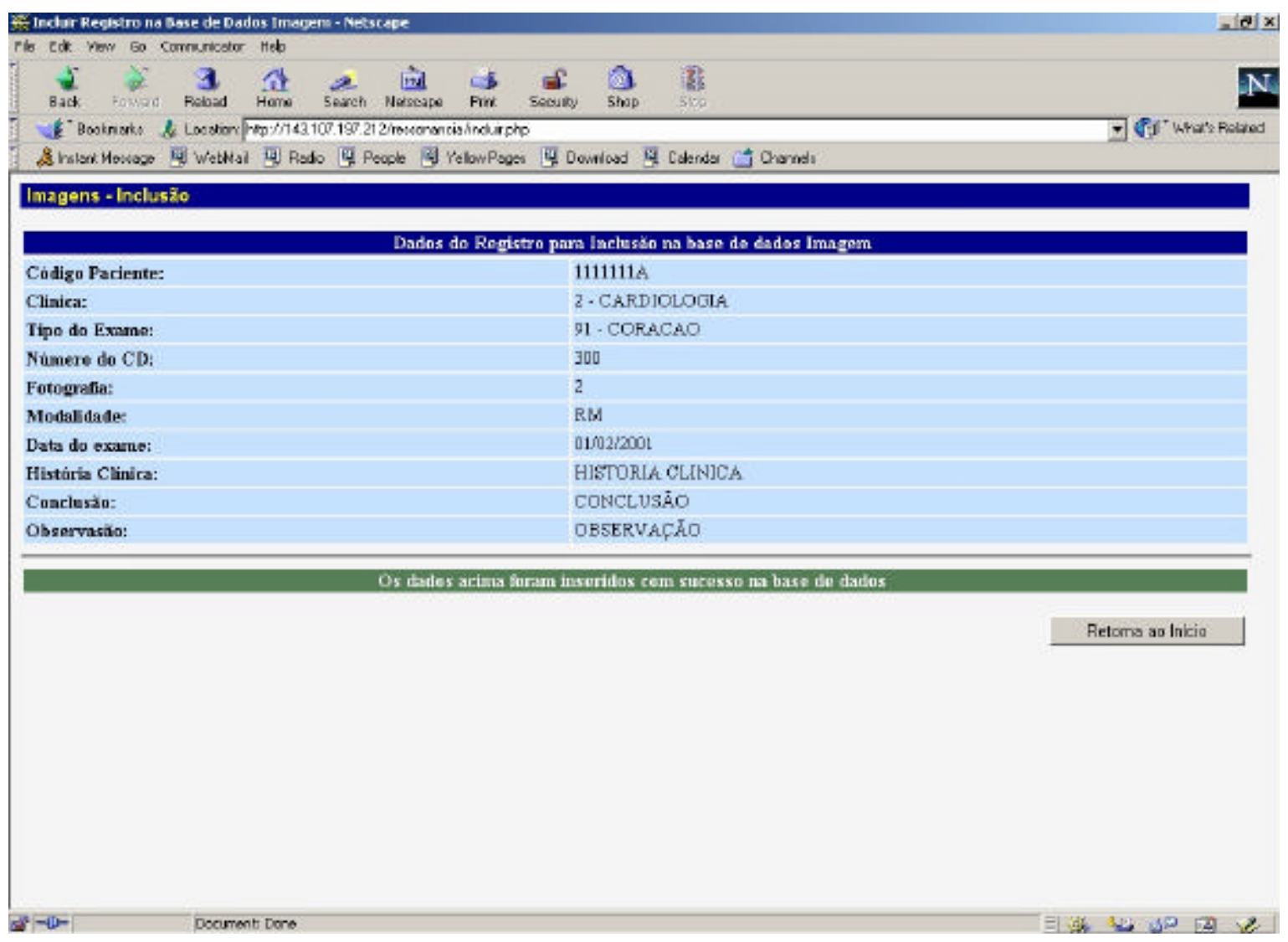

Figura 8.3 - Tela indicando que a inclusão foi realizada com sucesso.

No processo de inclusão todos os campos existentes devem ser preenchidos, caso isso não ocorra, a inclusão falha e o sistema informa ao usuário os campos que ficaram sem informação e obrigatoriamente devem ser preenchidos (Figura 8.4).

A proposta é que, os exames gerados atualmente sejam inseridos diretamente no Livro Eletrônico e as informações já existentes sejam inseridas posteriormente. Para que o sistema de vinculação além de retornar as imagens, possa informar o número do $\mathrm{CD}$ que contem as imagens, quando elas não estiverem “on-line”.

A consulta pode ser realizada através do Nome e/ou Sobrenome do paciente, pelo Número do Registro (RGHC) ou pela data da realização do exame. 
O Sistema retorna as informações necessárias para o usuário validar a consulta, tais como: Número do Exame (número seqüencial do Livro), Número do Registro do Paciente, Nome completo do Paciente, Data da Realização do Exame e o Número do CD, indicando onde as imagens estão armazenadas (Figura 8.5).

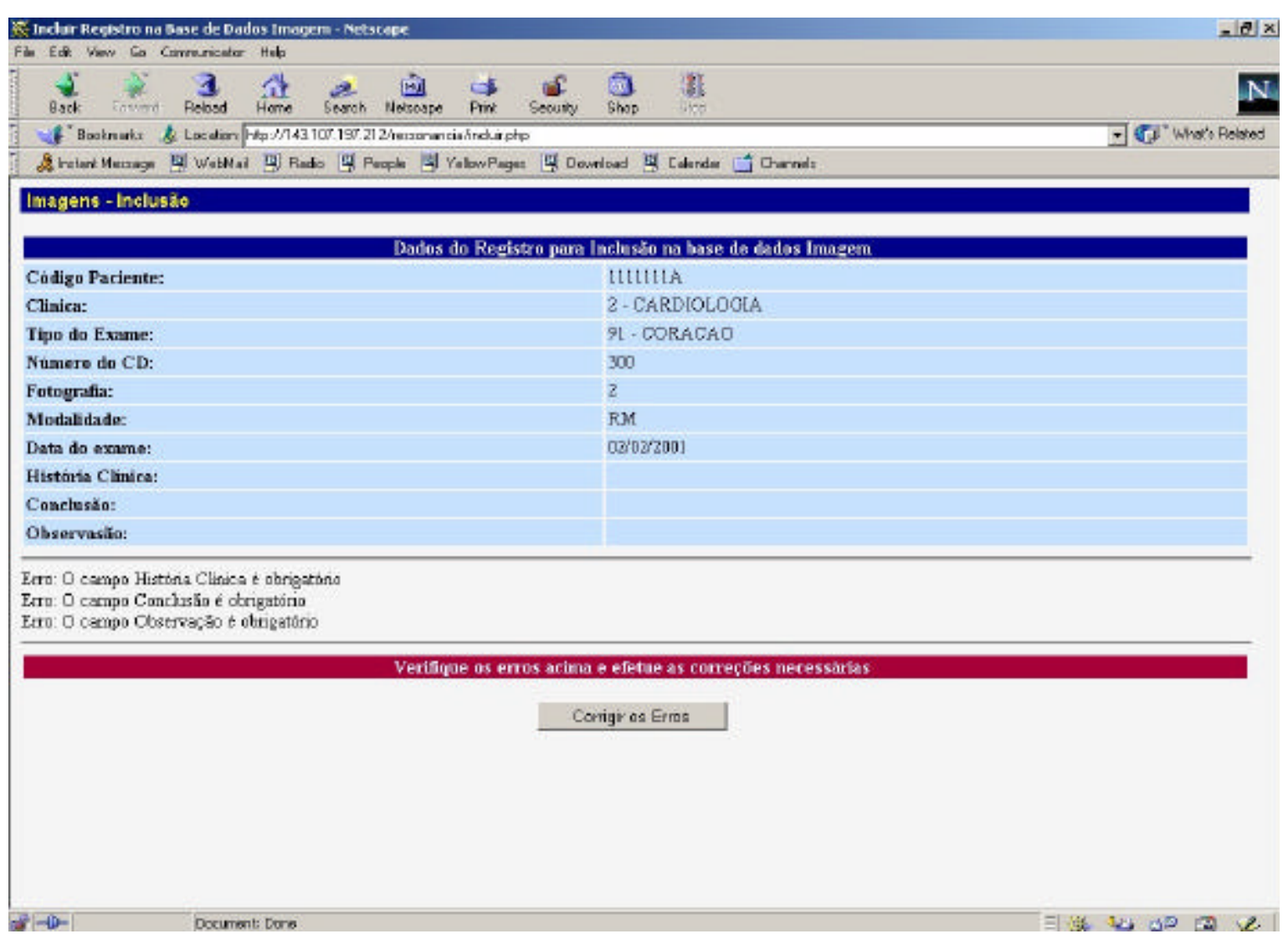

Figura 8.4 - Tela indicando que a inclusão não foi realizada, informando os campos em branco.

Esta Base de Dados também é utilizada pelo Sistema de Recuperação e Visualização das Imagens ("Laudo Web"). Quando ocorre uma consulta, o sistema busca na Base de Dados das Imagens as imagens do exame solicitado e caso essas imagens não sejam encontradas, outra busca é realizada na Base de Dados do Livro Eletrônico para que seja informado ao usuário o número do $\mathrm{CD}$ que contém as imagens do exame solicitado. 


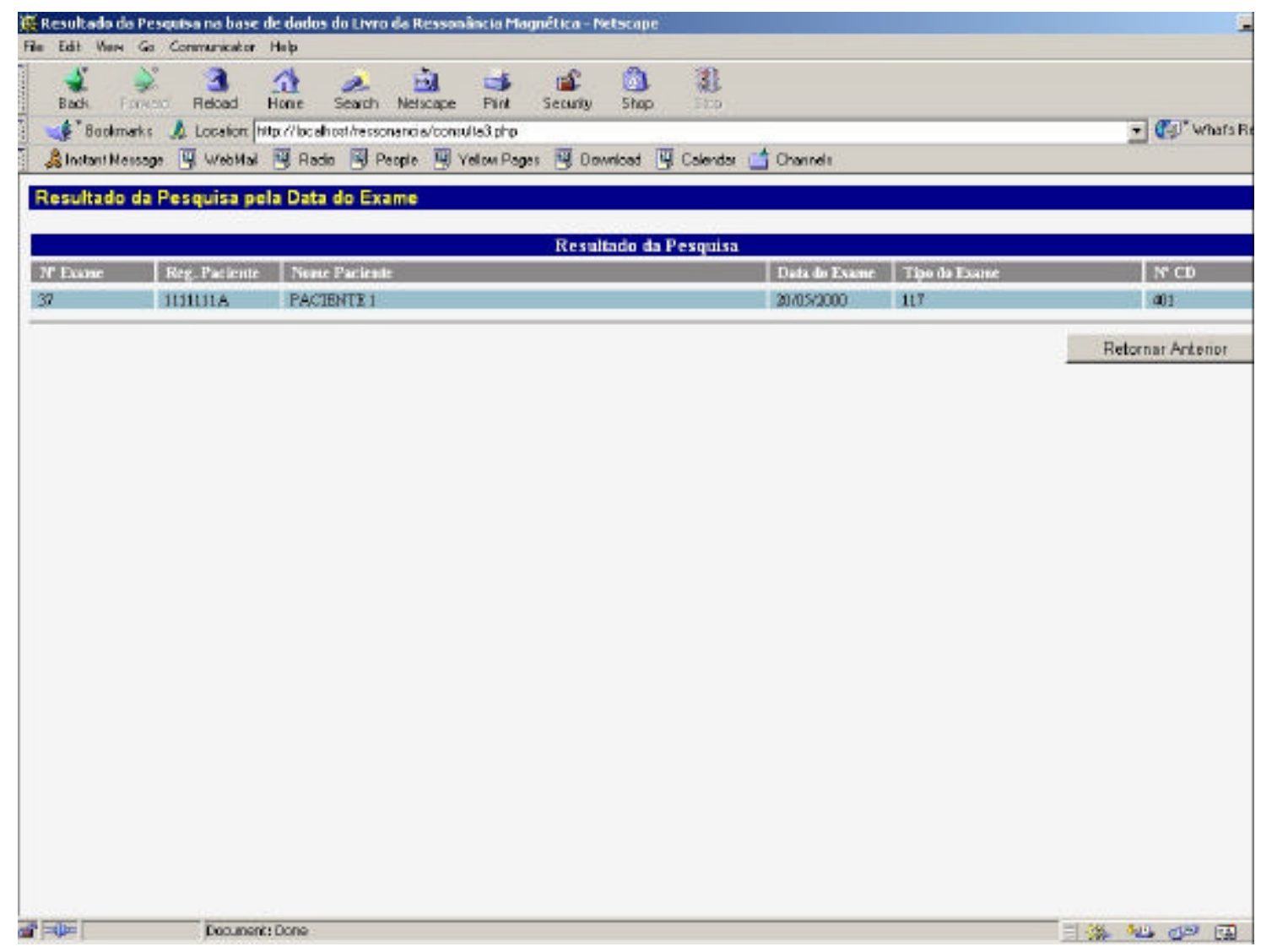

Figura 8.5 - Tela de resultado de uma consulta.

\subsection{Interface para leitura e abertura dos arquivos DICOM}

Foi desenvolvido um leitor para as imagens que estão no padrão DICOM 3.0 (Figura 8.6).

O leitor DICOM foi desenvolvido utilizando-se a linguagem $\mathrm{C}++$ "Builder". O sistema lê os arquivos que estão em $\mathrm{CD}$ ou no próprio $\mathrm{HD}$, extraindo as informações necessárias para a vinculação dos exames, armazenando essas informações no Banco de Dados responsável pelo gerenciamento das imagens. 


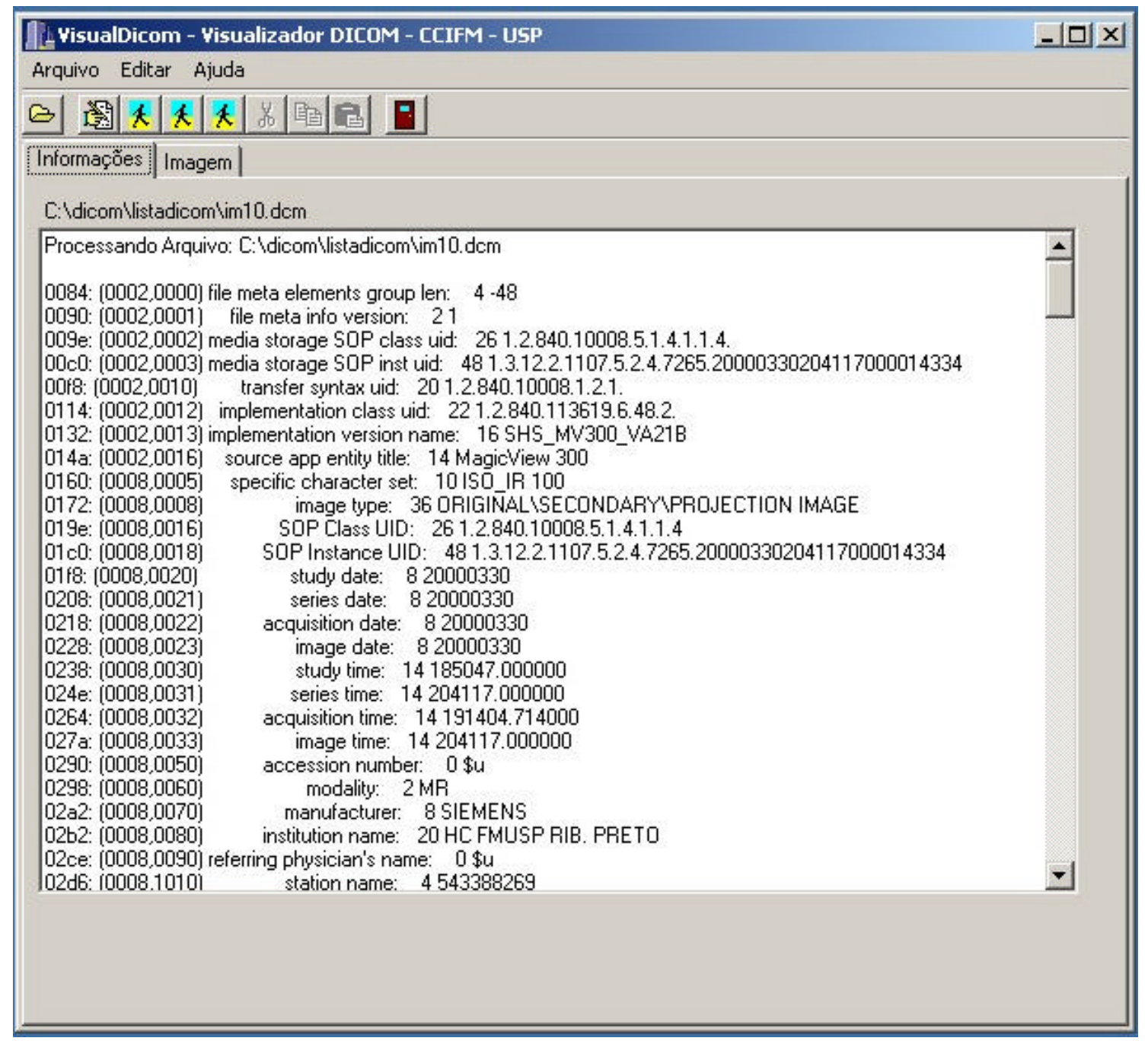

Figura 8.6 - Tela do Leitor DICOM e informações contidas na imagem.

\subsection{Interface de Recuperação e Visualização}

A Figura 8.7 apresenta a tela inicial do sistema, a qual permite ao usuário acessar o sistema através de um “Login” e uma Senha.

Após o usuário digitar seu "login" e sua senha, o sistema verifica se este usuário está autorizado a utilizar o sistema. Uma vez autorizado, o sistema informa o nome 
completo, hora inicial do acesso e o número IP ("Internet Protocol") do computador que ele está utilizando. Se o usuário não estiver autorizado, ele não terá acesso às consultas.

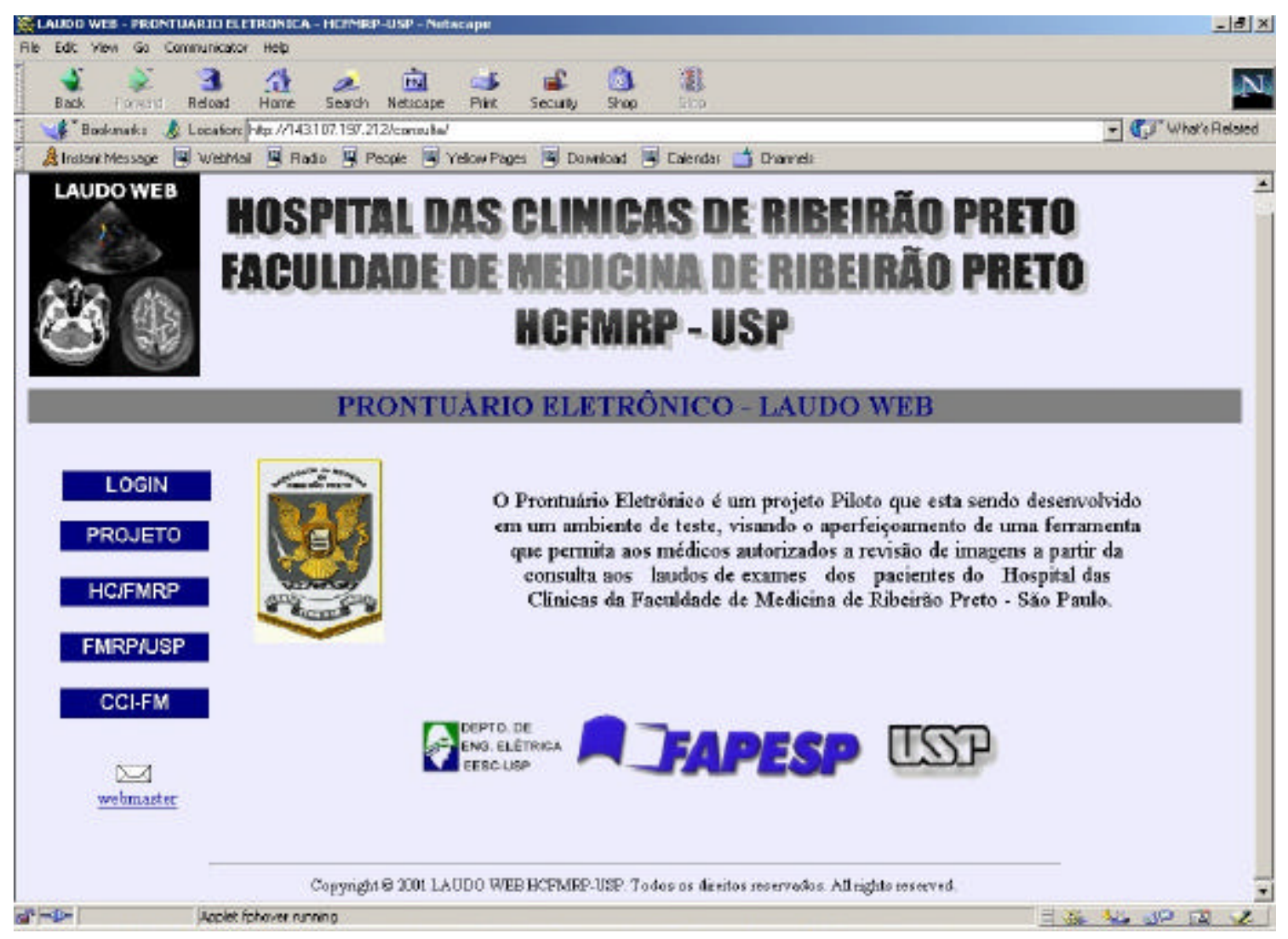

Figura 8.7 - Tela Inicial do "Laudo Web” (Sistema de Vinculação e Visualização de Imagens Médicas).

O sistema permite ao usuário autorizado, consultar as informações dos pacientes, seus exames e suas respectivas imagens, tanto de Tomografia Computadorizada, quanto de Ressonância Magnética já inseridas na Base de Dados do "Laudo Web".

As consultas podem ser feitas através do nome e/ou sobrenome do paciente ou através do seu número de matrícula no hospital (RGHC) (Figuras 8.8 e 8.9).

\footnotetext{
${ }_{5}^{5}$ IP - Internet Protocol ou protocolo internet. Conjunto de 32 bits que atribui o endereço de um computador em redes TCP/IP com o propósito de localizá-lo dentro da internet. É o protocolo da camada 3 de rede na arquitetura ISSO. Entre suas funções, proporciona uma conexão para protocolos de nível superior, assumindo funções como rastrear endereços de nós, rotas para envio de mensagens, reconhecimento de mensagens recebidas, além de se responsabilizar por localizar e manter o melhor caminho de tráfego na topologia da rede.
} 


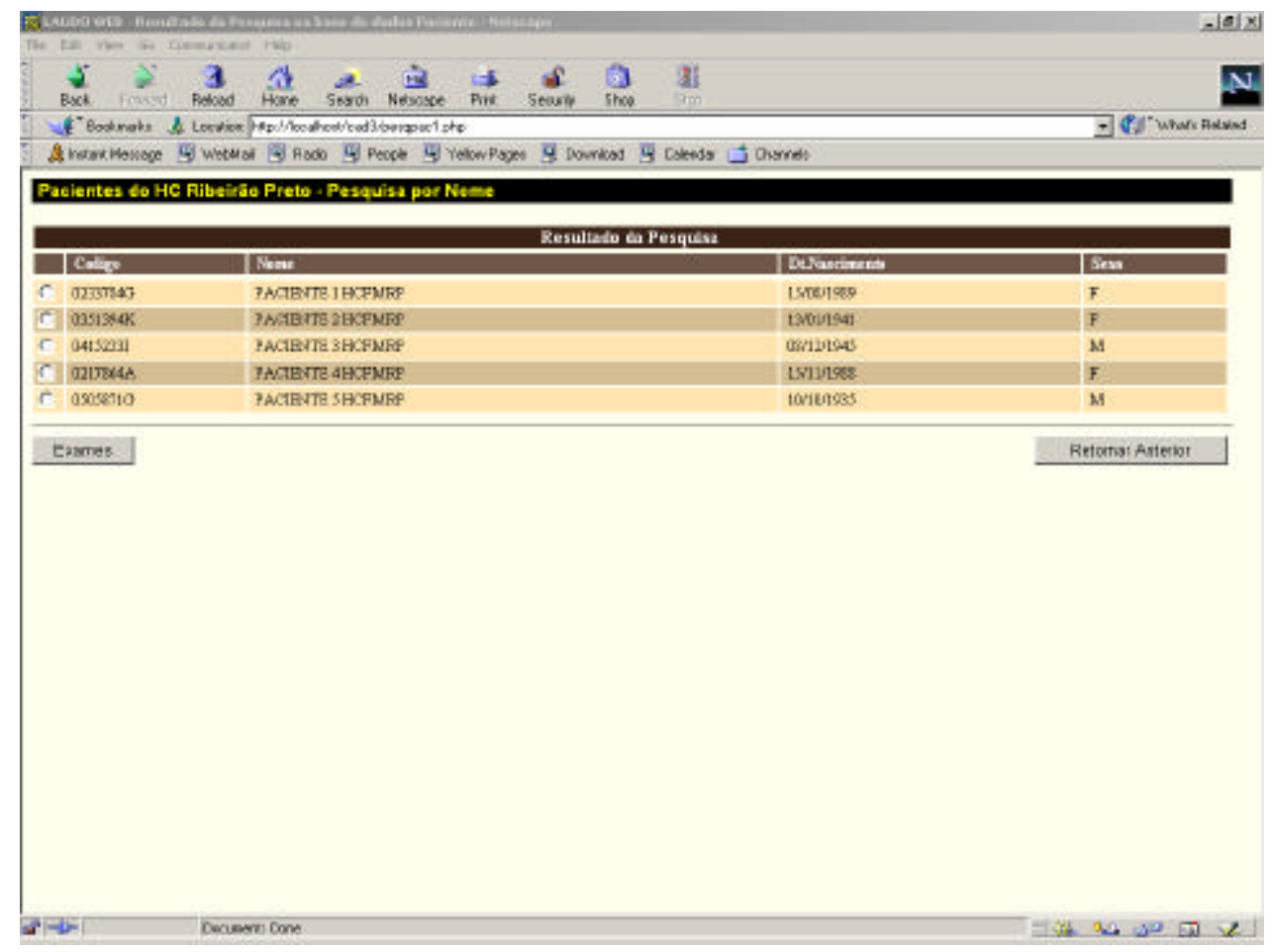

Figura 8.8 - Resultado de uma consulta por Sobrenome de paciente.

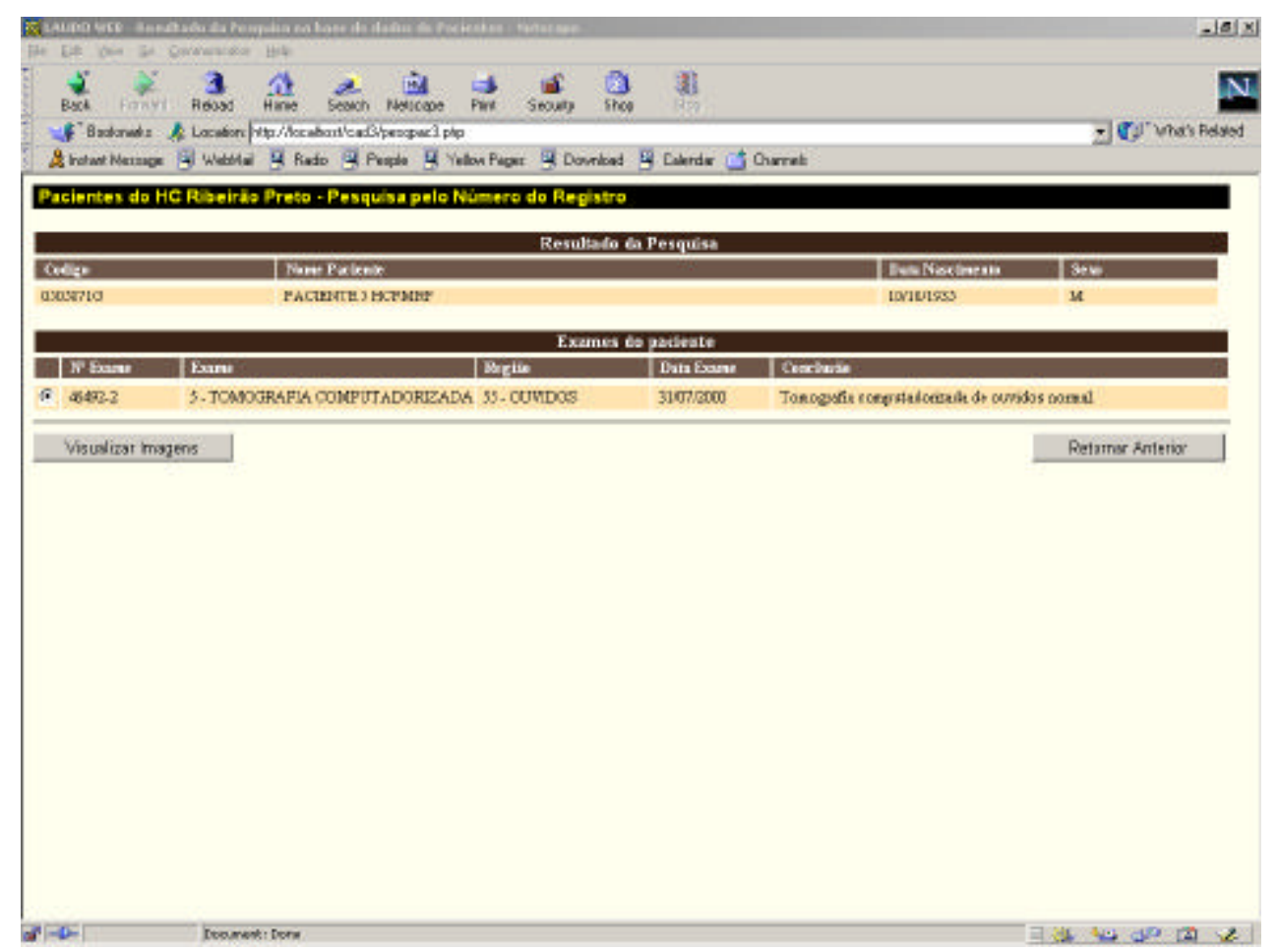

Figura 8.9 - Resultado de u ma consulta pelo número do Registro do paciente. 
Quando realizada uma consulta por Sobrenome o sistema retorna a lista de todos pacientes com este sobrenome, isso permite ao usuário selecionar o paciente desejado e visualizar os exames que este paciente possui. Esta opção foi implementada devido à possibilidade do usuário não possuir o número do registro do paciente ou seu nome completo.

$\mathrm{Na}$ consulta realizada através do número do registro, o sistema retorna as informações e os exames realizados pelo paciente através de uma única busca, isso também acontece quando se sabe o nome completo do paciente.

Quando o sistema não encontra o paciente desejado utilizando qualquer uma das opções apresentadas, uma mensagem é retornada ao usuário informando que não consta nenhuma informação referente ao paciente pesquisado.

A partir das informações do paciente e da lista dos exames realizados é possível acessar as imagens. Caso o usuário selecione algum exame em que a imagem ainda não esteja vinculada ao "Laudo Web" e se o exame não estiver cadastrado no Livro Eletrônico, o sistema retornará uma mensagem informando que as imagens encontram-se "off-line" e que não foi possível localizar o número do CD (Figura 8.10).

Os exames que possuem as imagens cadastradas na Base de Dados das imagens apresentam como resultado para o usuário as imagens do exame solicitado (Figura 8.11).

Após o retorno das imagens o usuário poderá verificar o laudo completo daquele exame, onde consta a suspeita clínica e o nome do médico responsável (Figura 8.12).

Caso as imagens não sejam encontradas é feita uma consulta na Base de Dados do Livro Eletrônico informando ao usuário o número do $\mathrm{CD}$ que contém as imagens, isso indica que as imagens estão "off-line" e o usuário deverá entrar em contato com os responsáveis pelo arquivo didático do hospital para obter as imagens (Figura 8.13). 


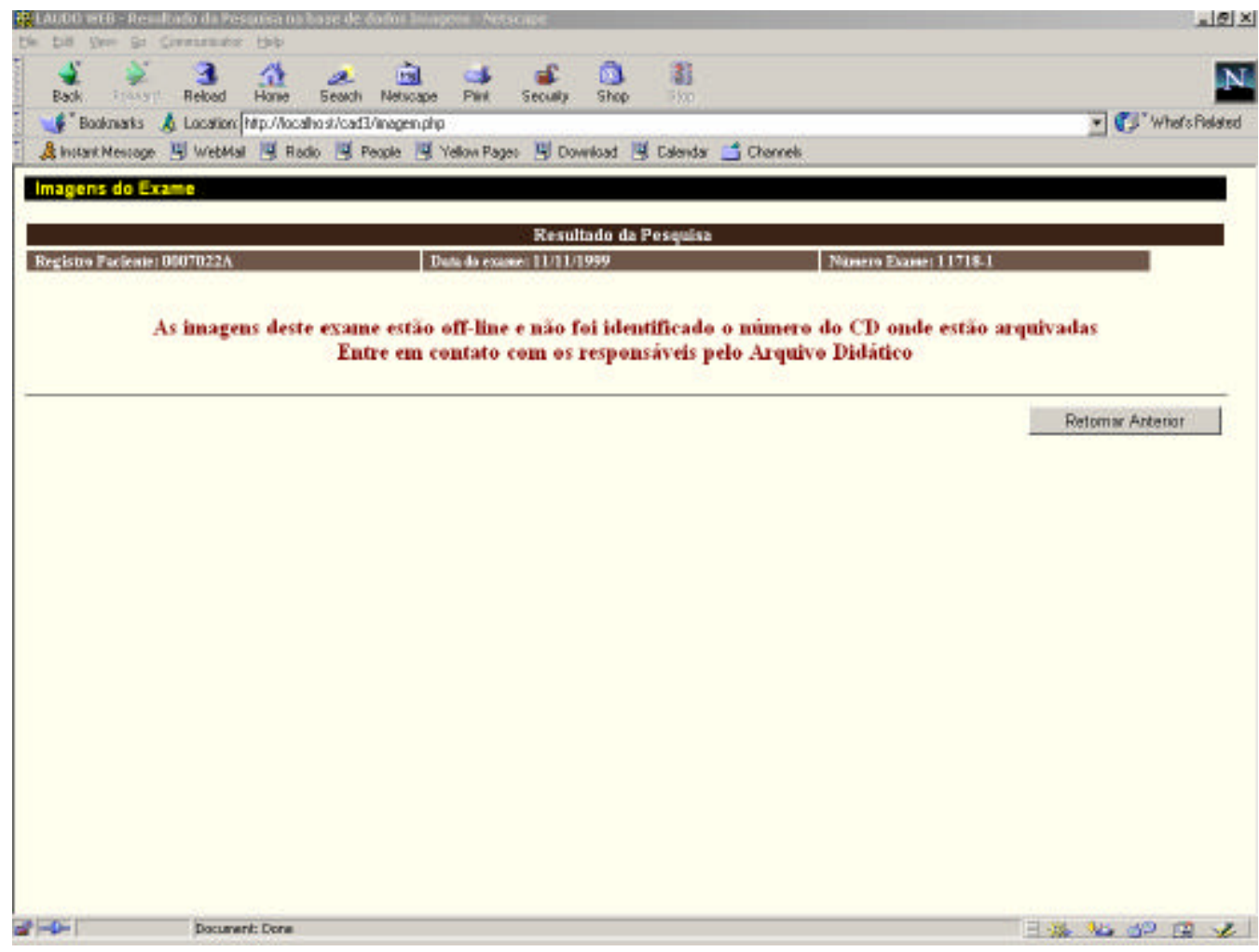

Figura 8.10 - Resultado de uma consulta onde às imagens não foram encontradas.

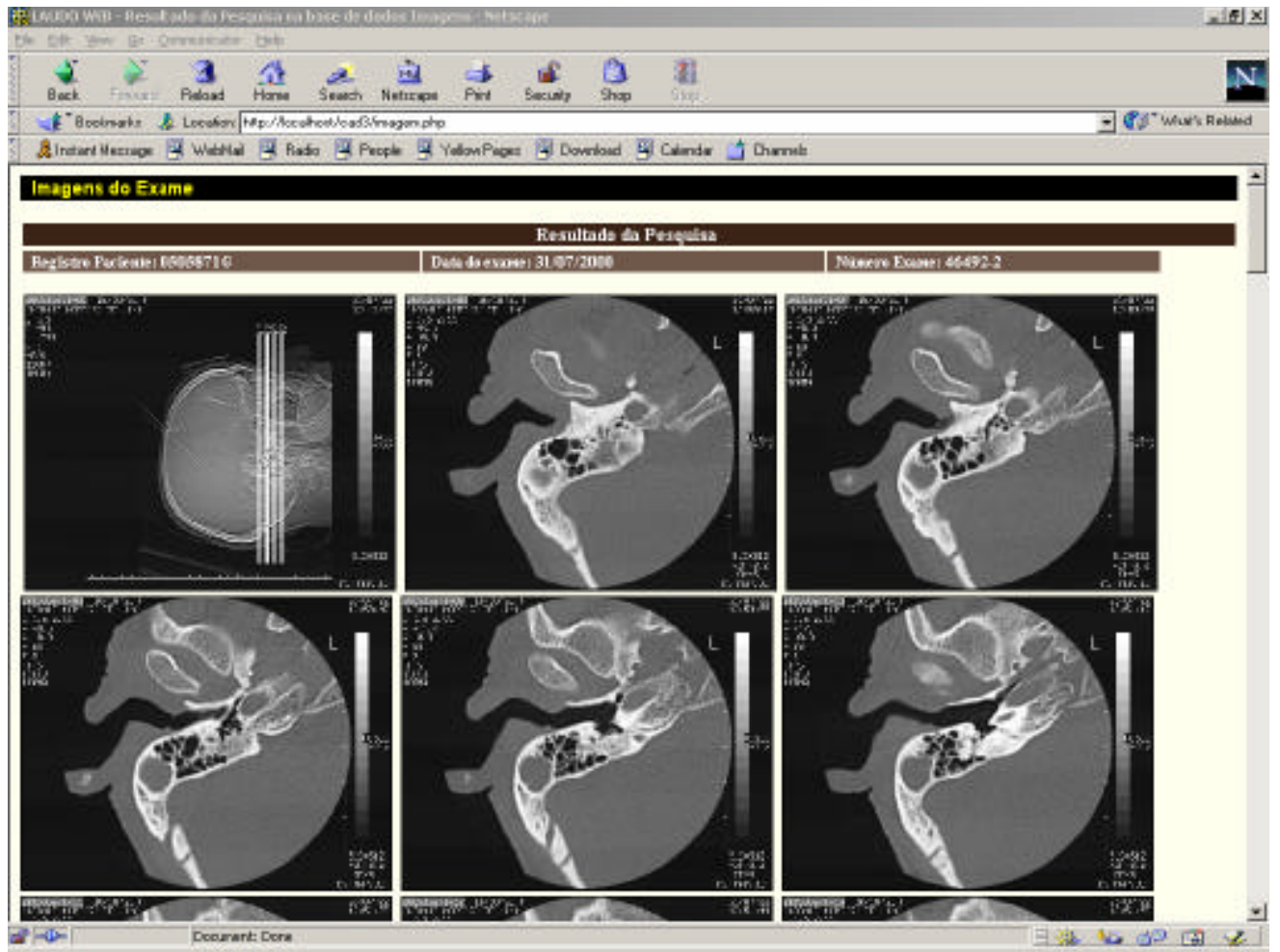

Figura 8.11 - Resultado de uma consulta onde às imagens foram retornadas. 


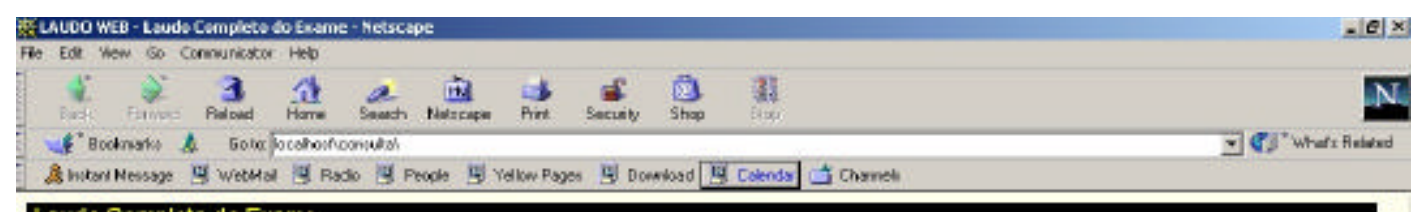

\section{Laudo Completo do Exame}

\begin{tabular}{|c|c|}
\hline \multicolumn{2}{|r|}{ Resultado ds Peserisa } \\
\hline Regigtro Paciente: 0505871G & Data do exame: $31,07 / 2000$ \\
\hline \multicolumn{2}{|l|}{ Suspeita Clinica } \\
\hline Laudo Completo & 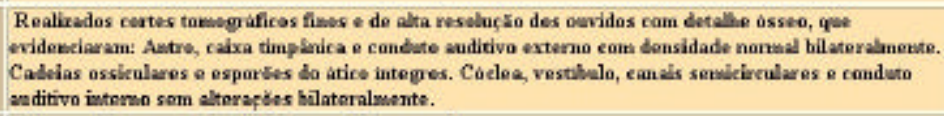 \\
\hline Conchusāo & Tamografia compatadorizada de ouvidos nonnal. \\
\hline Médico Responsável & Dr.(a) MEDICO RESPONSAVEL \\
\hline
\end{tabular}

Figura 8.12 - Resultado da consulta aos laudos completos.

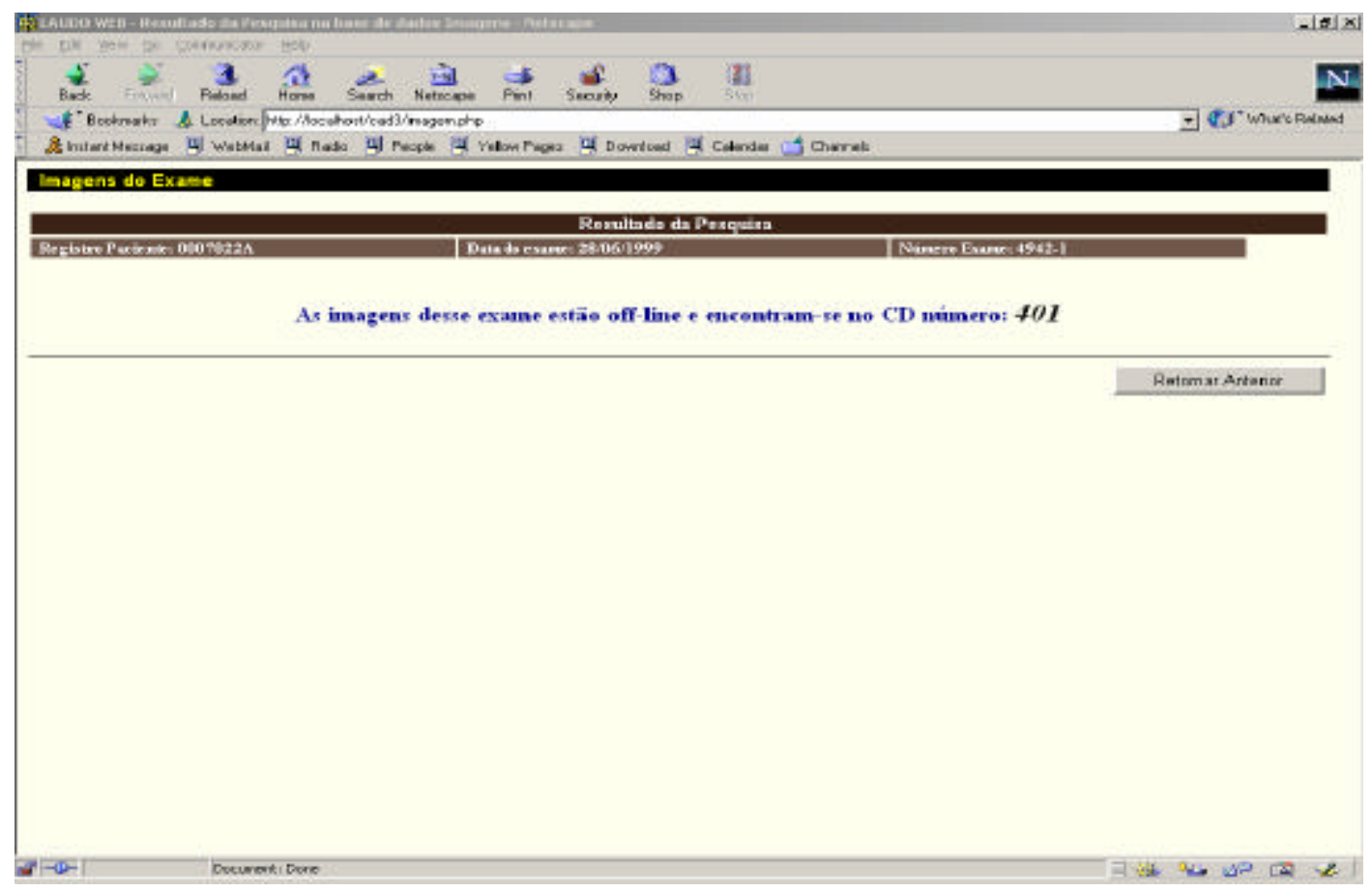

Figura 8.13 - Resultado da consulta as imagens onde o número do CD foi retornado. 


\section{8 “Applet Java” Visualizador DICOM}

Para que as imagens no formato DICOM fossem visualizadas no "browser" foi desenvolvido um "applet" utilizando a linguagem de programação Java. O "applet" possibilita a visualização das imagens, incluindo funções para ajuste de brilho e contraste (Figura 8.14).

Para seu desenvolvimento foram consideradas as variações do padrão DICOM, como exemplo, se o arquivo é "implícito", "explicito", "BigEndian" ou "LittleEndian", para tornar o sistema genérico e capaz de ler e abrir imagens geradas por diferentes fabricantes, pois o padrão DICOM possui algumas variações de leitura e gravação, sendo que cada fabricante implementa da maneira que lhe convém. O "applet" permite visualizar as imagens de Tomografia Computadorizada e Ressonância Magnética Nuclear e de outras modalidades, desde que as imagens estejam em "gray-scale".

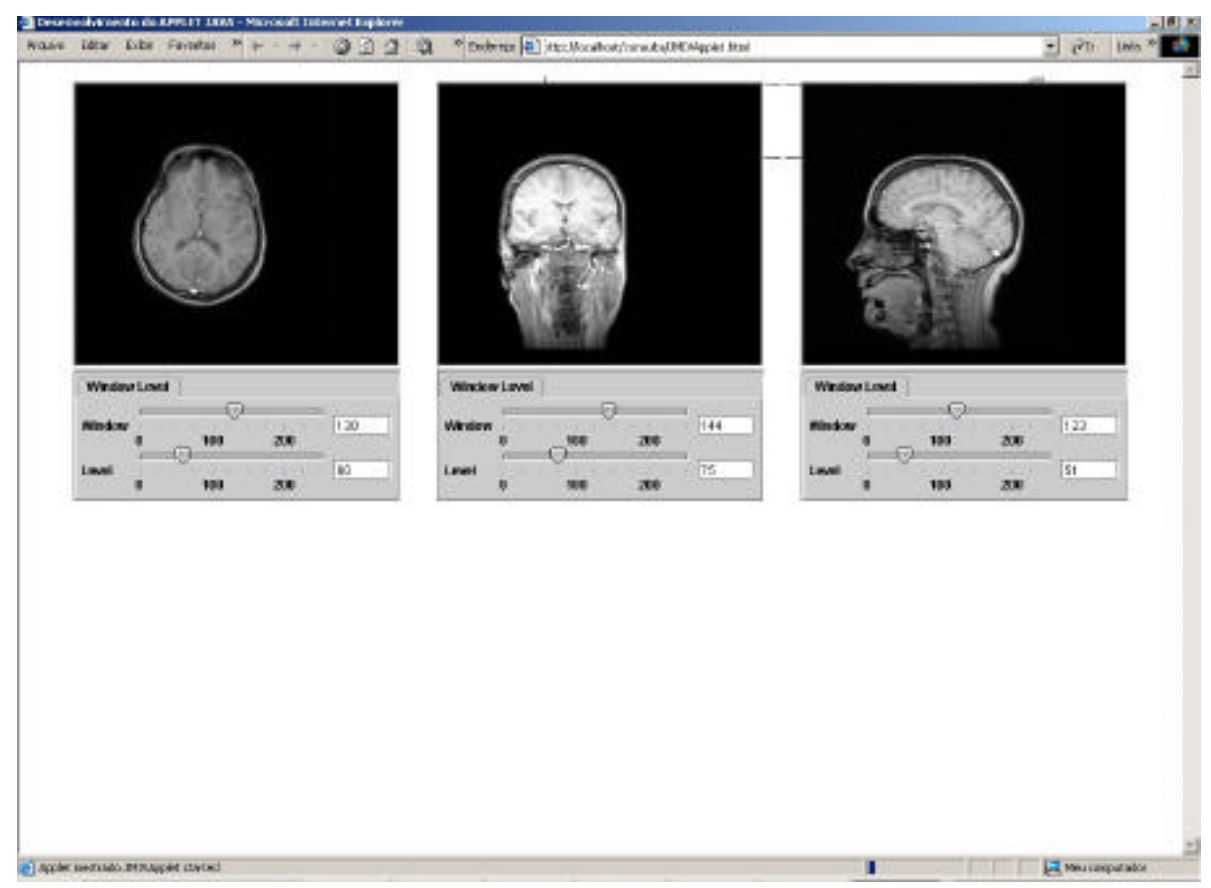

Figura 8.14 - Visualizador de imagens DICOM. 


\section{Capítulo 9}

\section{Resultados Obtidos e Discussão}

Neste capítulo são apresentados e descritos os resultados e discussões inerentes ao trabalho.

Para a maioria dos sistemas de indexação e recuperação de dados, tempo e espaço requerido são usualmente as métricas utilizadas para avaliação do sistema [HONDA-2001].

Neste trabalho, além do desempenho e precisão da indexação e recuperação, avaliamos a qualidade das imagens exibidas e a viabilidade do sistema aos médicos, dessa maneira temos uma avaliação quantitativa associada a uma qualitativa.

\subsection{Recuperação das Imagens}

A avaliação de desempenho é fundamental para qualquer sistema de busca e recuperação de imagens. Para tanto realizamos algumas consultas, utilizando exames de Ressonância Magnética (padrão DICOM) e de Tomografia Computadorizada (JPEG).

Os resultados das consultas são distintos, dado ao fato que cada exame possui um número diferente de imagens, que depende da estrutura anatômica e do tipo de modalidade de aquisição.

Comumente os exames de Ressonância Magnética possuem mais imagens do que os exames de Tomografia Computadorizada. 
Para a consulta 1, selecionamos aleatoriamente um exame de Tomografia Computadorizada de crânio. O laudo desse exame indica a existência de uma patologia, esse exame é formado por 30 imagens, essa informação foi obtida do CD que armazena as imagens desse exame.

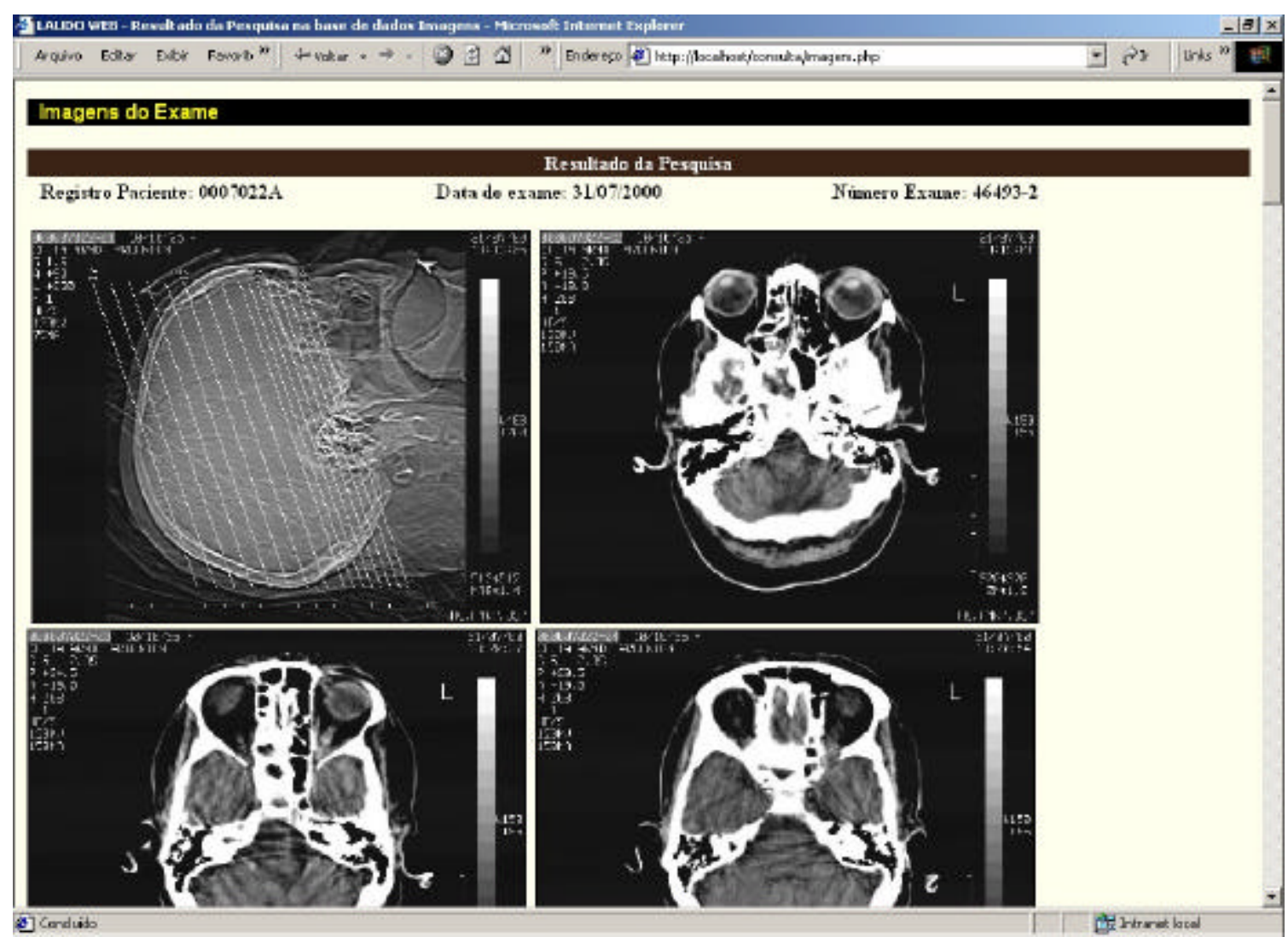

Figura 9.1 - Resultado da Consulta 1.

A Figura 9.1 apresenta as imagens resultantes da consulta 1, o sistema retornou 30 imagens e demorou 21 segundos e 25 milésimos para conclui-la.

Para a consulta 2, selecionamos aleatoriamente um exame de Tomografia Computadorizada de abdome, o laudo desse exame indica a existência de uma patologia, 
esse exame é formado por 172 imagens, essa informação foi obtida do CD que armazena as imagens desse exame.

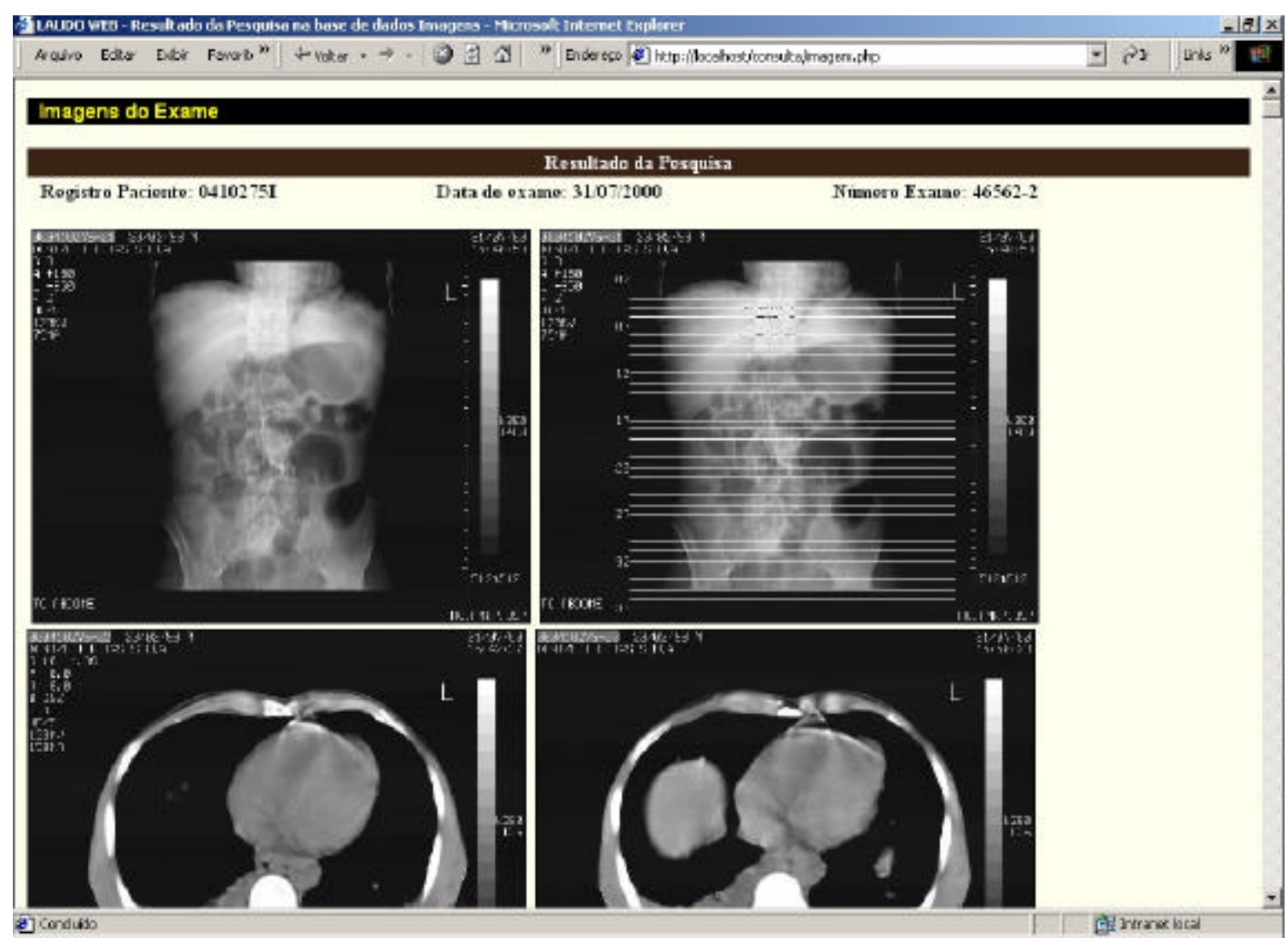

Figura 9.2 - Resultado da Consulta 2.

A Figura 9.2 apresenta as imagens resultantes da consulta 2, o sistema retornou 172 imagens e demorou 1 minuto, 15 segundos e 07 milésimos para concluir a consulta.

Para a consulta 3, selecionamos aleatoriamente um exame de Ressonância Magnética de coluna cervical, o laudo desse exame indica que não há patologia (exame normal), esse exame é formado por 171 imagens, essa informação foi obtida do CD que armazena as imagens desse exame. 


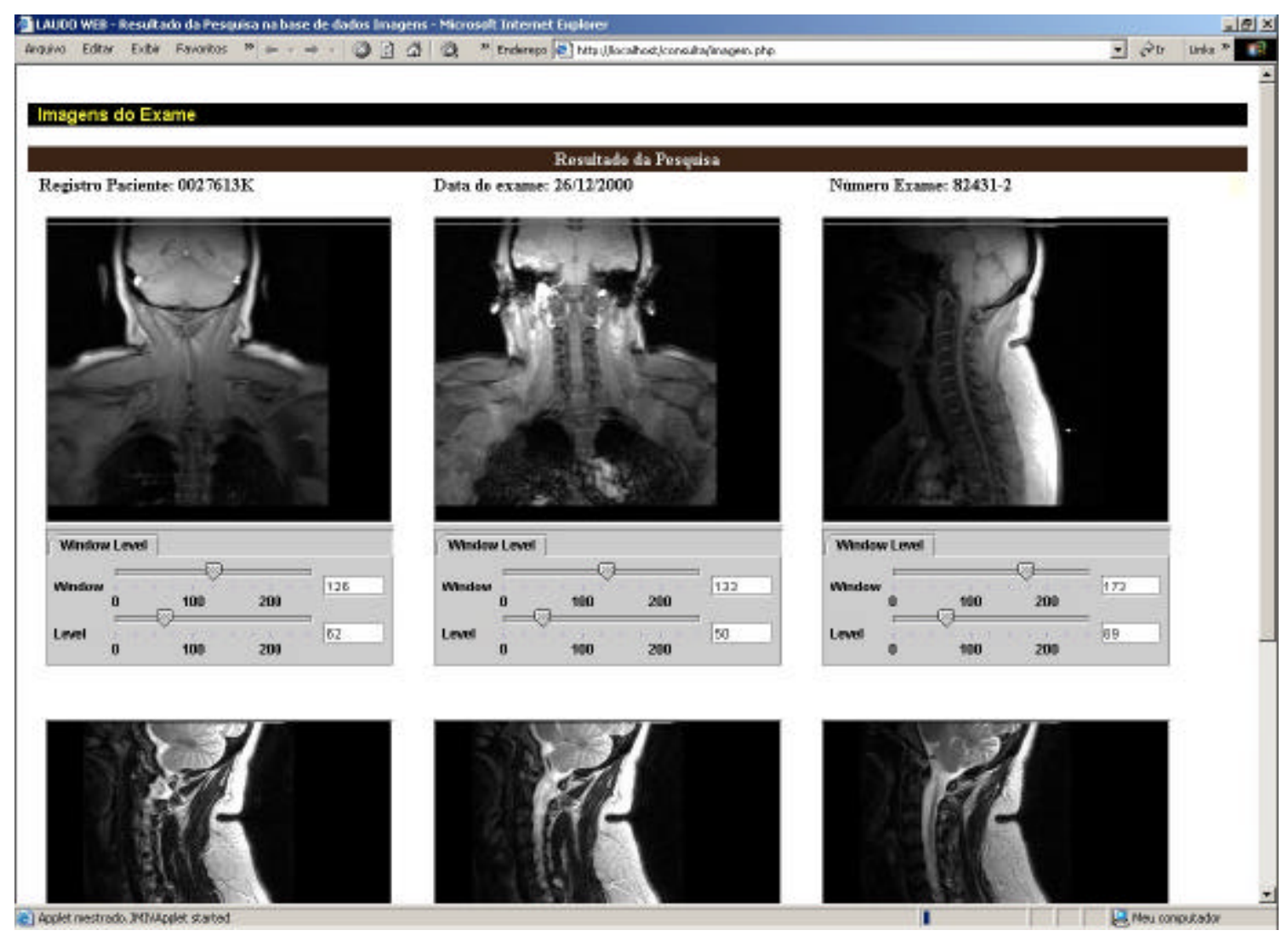

Figura 9.3- Resultado da Consulta 3.

A Figura 9.3 apresenta as imagens resultantes da consulta 3, o sistema retornou 171 imagens e demorou 2 minutos, 30 segundos e 72 milésimos para concluir a consulta.

Para a consulta 4, selecionamos aleatoriamente um exame de Ressonância Magnética de colangioressonância, o laudo desse exame indica que não há patologia (exame normal). O exame é formado por 238 imagens, essa informação foi obtida do CD que armazena as imagens desse exame.

A Figura 9.4 apresenta as imagens resultantes da consulta 4, o sistema retornou 238 imagens e demorou 3 minutos, 25 segundos e 98 milésimos para concluir a consulta. 


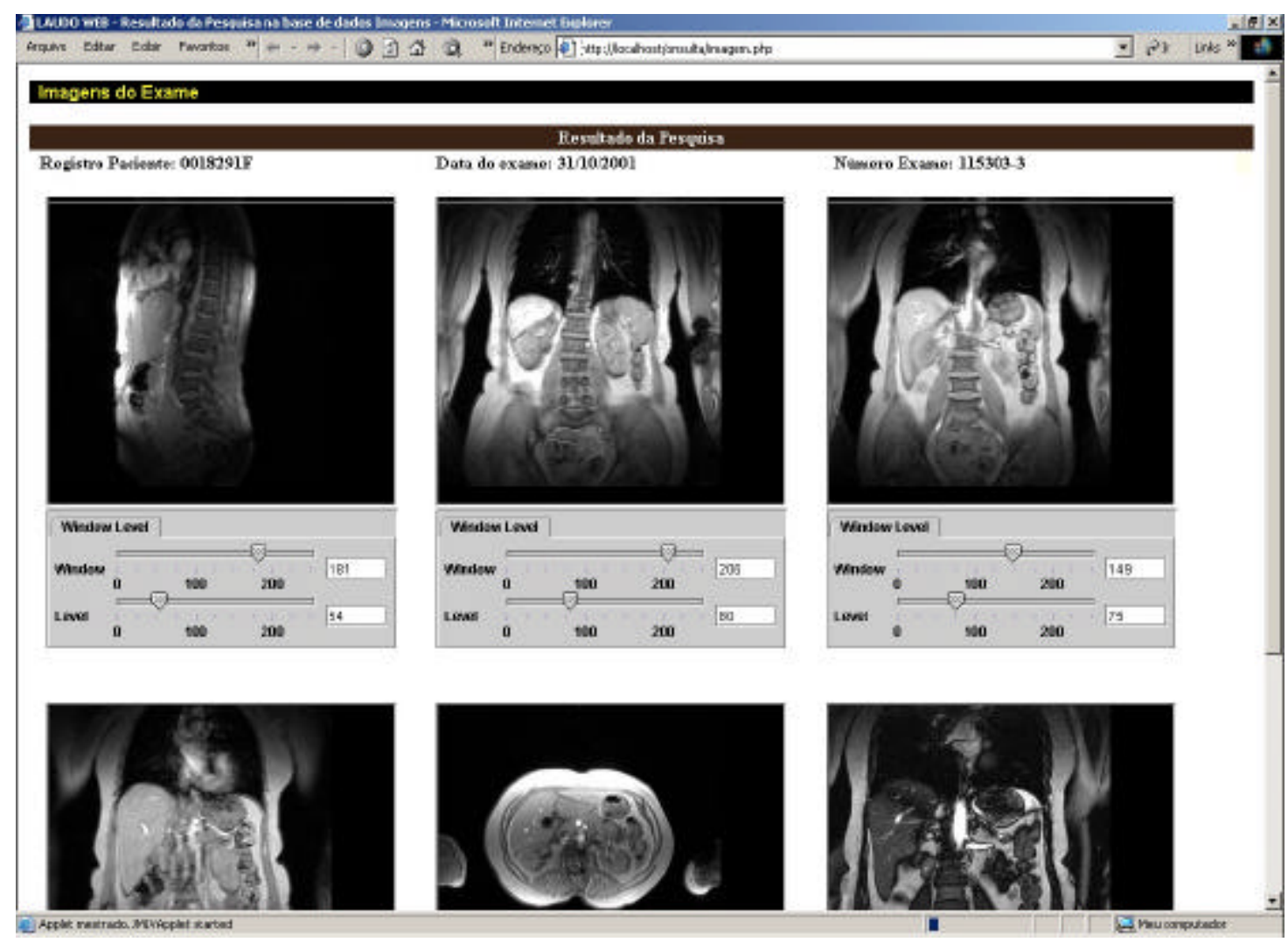

Figura 9.4- Resultado da Consulta 4.

As consultas foram realizadas em um PC Intel Pentium III $600 \mathrm{MHz}$, com $512 \mathrm{MB}$ de memória RAM e monitor de vídeo de 17", utilizando o "browser" "Internet Explorer 5.5” em uma rede “ethernet” 10/100 Mbps.

$\mathrm{Na}$ tabela 9.1 apresentamos os resultados obtidos com as consultas realizadas para avaliação de desempenho e precisão.

\begin{tabular}{|l|c|c|c|}
\hline Modalidade & Número de Imagens & Tempo de Espera & Precisão \\
\hline Tomografia Computadorizada & 30 & $21 \mathrm{~s} 25 \mathrm{~m}$ & $100 \%$ \\
\hline Tomografia Computadorizada & 172 & $1 \mathrm{~min} .15 \mathrm{~s} 07 \mathrm{~m}$ & $100 \%$ \\
\hline Ressonância Magnética & 171 & 2 min. $30 \mathrm{~s} 72 \mathrm{~m}$ & $100 \%$ \\
\hline Ressonância Magnética & 274 & 3 min. $25 \mathrm{~s} 98 \mathrm{~m}$ & $100 \%$ \\
\hline
\end{tabular}

Tabela 9.1 - Resultado Obtidos. 
Com os resultados obtidos podemos destacar que o trabalho apresenta um bom desempenho e uma boa precisão. Segundo Sheng et al., o tempo ideal de resposta de um sistema de recuperação de imagens médicas deve ser inferior ao tempo necessário para retirar manualmente um filme de uma pasta de arquivo [SHENG-1990].

O bom desempenho do sistema pode ser avaliado também, considerando que atualmente são necessários em média dois dias para que o médico tenha acesso as imagens dos exames, isso se o $\mathrm{CD}$ que contém as imagens do exame desejado não estiver emprestado para outra pessoa.

Outro resultado positivo que pode ser destacado é que com o sistema será evitada a perda de cópias originais das imagens durante o transporte pelo hospital, a maioria das imagens fica a disposição dos médicos, com a possibilidade de acesso simultâneo de vários usuários, o que antes não era possível.

Para os testes utilizamos uma base de dados com 50 exames, totalizando 5.691 imagens.

\subsection{Avaliação pelos médicos especialistas}

Levando em consideração que o médico será o maior beneficiado com esse trabalho, optamos também por fazer uma quantificação do desempenho a partir da avaliação qualitativa do médico.

Para isso pedimos que alguns médicos do Centro de Ciências das Imagens do Hospital das Clínicas de Ribeirão Preto utilizassem e avaliassem o sistema de recuperação e visualização de imagens, seguindo os critérios da tabela 9.2. 


\begin{tabular}{|c|l|}
\hline Índice & \multicolumn{1}{|c|}{ Descrição } \\
\hline 1 & Muito Ruim \\
\hline 2 & Ruim \\
\hline 3 & Satisfatório \\
\hline 4 & Bom \\
\hline 5 & Muito Bom \\
\hline
\end{tabular}

Tabela 9.2 - Índices para avaliação do Sistema.

Os itens a serem avaliados pelos médicos especialistas são; interface do sistema, tempo de retorno dos exames, tempo de retorno das imagens, qualidade das imagens, facilidade de navegação e ajuste da imagem.

O sistema foi avaliado por dois médicos, que apresentaram os seguintes resultados, conforme a tabela 9.3.

\begin{tabular}{|l|c|c|}
\hline \multicolumn{1}{|c|}{ Item } & Médico 1 & Médico 2 \\
\hline Interface do sistema & 4 & 5 \\
\hline Tempo de retorno dos exames & 3 & 5 \\
\hline Tempo de retorno das imagens & 3 & 5 \\
\hline Qualidade das imagens & 4 & 4 \\
\hline Facilidade de navegação & 4 & 5 \\
\hline Ajuste da imagem & 3 & 3 \\
\hline
\end{tabular}

Tabela 9.3 - Avaliação segundo os médicos especialistas.

A partir da avaliação individual de cada médico especialista calculamos uma média que é apresentada na tabela 9.4.

\begin{tabular}{|l|c|l|}
\hline \multicolumn{1}{|c|}{ Item } & Média & \multicolumn{1}{c|}{ Conclusão Final } \\
\hline Interface do sistema & 5 & Muito Bom \\
\hline Tempo de retorno dos exames & 4 & Bom \\
\hline Tempo de retorno das imagens & 4 & Bom \\
\hline Qualidade das imagens & 4 & Bom \\
\hline Facilidade de navegação & 5 & Muito Bom \\
\hline Ajuste da imagem & 3 & Satisfatório \\
\hline
\end{tabular}

Tabela 9.4 - Resultado final da avaliação pelos médicos especialistas. 
Como resultado da avaliação qualitativa dos médicos especialistas podemos destacar que o sistema é considerado bom, sendo sugerido pelos médicos adicionar algumas ferramentas para melhorar o ajuste das imagens tais como: zoom, segmentação, binarização e outras técnicas utilizadas no processamento de imagens.

\subsection{Padronização na realização do exame}

Outro resultado importante obtido através deste trabalho é que para a implementação deste projeto "piloto" no hospital será necessário criar junto aos médicos um padrão de inserção das informações na realização do exame. Isso se faz necessário devido à falta de informações essenciais não preenchidas.

Em vários arquivos de imagens encontramos informações no cabeçalho que dificultam a vinculação de alguns exames e a maioria dos exames não contém o número do exame no arquivo imagem, que seria o identificador único para cada exame (campo chave primária). Para solucionar esse problema quando realizada uma consulta, busca-se o número do exame, caso esse não seja encontrado é feita uma associação com o número da matrícula do paciente (RGHC), mais a data da realização do exame, mais a modalidade (CT, RM ou outra) para retornar as imagens, mas dessa forma não podemos garantir que o sistema retornará as imagens corretas do exame, pois o mesmo paciente pode fazer no mesmo dia mais de um exame de uma mesma modalidade.

A partir desse resultado foi proposto aos médicos que estabeleçam um padrão ou protocolo para inserir as informações do paciente na realização do exame, assim definimos que as informações: data de realização do exame, nome do paciente, número do registro do 
paciente (RGHC), número do exame, modalidade que realizou o exame, são informações essenciais para uma posterior vinculação e recuperação das imagens.

O padrão DICOM estabelece que o número do exame seja informado na "tag" "Acession Number", pois esta é utilizada pelos servidores DICOM como o identificador único de cada exame e esse número é utilizado para organizar as imagens. Sendo assim foi proposta esta solução para os usuários, mas lembramos que existe um período de adequação ao novo protocolo sugerido. Acreditamos que num futuro próximo poderemos recuperar todos os exames com $100 \%$ de consistência. 


\section{Capítulo 10}

\section{Conclusões}

\subsection{Conclusões Finais}

A conclusão deste trabalho é feita considerando os dois objetivos principais: vincular as imagens com seus laudos e o desenvolvimento da interface de visualização das imagens de Tomografia Computadorizada e Ressonância Magnética.

O primeiro objetivo foi atingido totalmente, sendo que foi desenvolvido e implementado o módulo para inserção das informações necessárias para vincular os exames de Tomografia Computadorizada que estão no formato JPEG, bem como o algoritmo de leitura e armazenamento na Base de Dados das informações dos pacientes, necessárias para a vinculação extraídas automaticamente das imagens que estão no padrão DICOM.

O segundo objetivo também foi realizado através da implementação do "applet Java” para visualização das imagens que estão no padrão DICOM, porém poderia ter sido implementado a esse visualizador algumas ferramentas adicionais para ajuste da imagem, além do ajuste de brilho e contraste que foram implementados.

Concluímos que este trabalho tornará a consulta aos exames e suas imagens, mais rápida e segura, considerando que o médico terá acesso global ao histórico do paciente e desde que ele esteja autorizado não precisará se deslocar até o local de armazenamento das imagens para visualiza-las. 


\subsection{Sugestões para Trabalhos Futuros}

Nessa seção apresentamos algumas sugestões para a continuidade deste trabalho. Tais sugestões provem da experiência adquirida no desenvolvimento do sistema, bem como das dificuldades encontradas.

1. Desenvolvimento de algoritmo para que o "applet" Java desenvolvido, possa ler e visualizar imagens coloridas;

2. Desenvolvimento de algoritmo para visualização 3D das imagens de Ressonância Magnética, através do "applet”.

3. Desenvolvimento e Implementação de um Servidor de Imagens DICOM, para aquisição, armazenamento e gerenciamento das imagens;

4. Desenvolvimento de algoritmos para gerenciamento das imagens na estrutura “on-line”, “nearline” e off-line”;

5. Estudo e estruturação de uma infra-estrutura de rede adequada para instalação de um Mini-PACS;

6. Desenvolvimento e implementação de um Mini-PACS;

7. Desenvolvimento de algoritmos para indexação e recuperação de imagens a partir da similaridade de seu conteúdo pictórico, podendo ser utilizada como uma outra ferramenta de acesso às informações. 


\section{Referências Bibliográficas}

[ABRAHÃO-2000]

ABRAHÃO, M.T.F.; AMORIN, M.F. A Utilização do HL7 na Comunicação de

Sistemas de Informação em Saúde. Anais do XVII Congresso Brasileiro de Engenharia Biomédica, pp. 1128-1132, 2000.

[ADELHARD-1999]

ADELHARD， K.;NISSEN-MEYER， S., PISTITSCH， C., FINK， U.; REISER, M. Functional Requirements for a HIS-RIS-PACS-Interface Design Including Integration of "Old" Modalities. Methods of Information in Medicine, vol. 38, pp. 1-8, 1999.

[ANDRIOLE-1999]

ANDRIOLE, K.P. Anatomy of Picture Archiving and Communications Systems: Nuts and Bolts - Image Acquisition: Getting Digital Images From Imaging Modalities. Journal of Digital Imaging, vol. 12, no 2, suppl. 1, pp. 216-217, maio, 1999.

[ARENSON-2000]

ARENSON, R.L.; ANDRIOLE K.P.; AVRIN D.E.; GOULD R.G. Computers in Imaging and Health Care: Now and in the Future. Journal of Digital Imaging, vol. 13, $\mathrm{n}^{\mathrm{o}} 4$, pp. 145-156, novembro, 2000. 
[AZEVEDO-MARQUES-2000]

AZEVEDO-MARQUES, P.M.; SANTOS A.C.; JÚNIOR, J.E.; GOES W.M.; CASTRO

C.R.; TRAD C.S. Implantação de um sistema de informação em Radiologia em Hospital Universitário. Radiologia Brasileira, vol. 33, pp. 155-160, 2000.

[BAKKER-1991]

BAKKER, A.R. HIS and RIS and PACS. Nato Ansi Series - Picture Archiving and Communications System (PACS) in Medicine, Springer-Verlag, pp. 157-162, 1991.

[BEHLEN-2000]

BEHLEN, F.M; SAYRE, R.E.; WELDY, J.B.; MICHAEL, J.S. 'Permanent" Records: Experience With Data Migration in Radiology Information System and Picture Archiving and Communication System Replacement. Journal of Digital Imaging, vol. 13, no 2 , suppl. 1, pp. 171-174, maio, 2000.

[BEIRD-1999]

BEIRD, L.C. The importance of a Picture Archiving and Communications System (PACS) Manager for Large-Scale PACS Installations. Journal of Digital Imaging, vol. 12, no 2, suppl. 1, pp. 37, maio, 1999.

[BENNETT-1999]

BENNETT, W.F.; SPIGOS, D.G.; TZALONIKOU, M.T.; TERELL, J.E.; AUGUSTYN, M. A. Web-based Viewing of Picture Archiving and Communications Systems Images 
- Part II: Optimal Personal Computer Configuration. Journal of Digital Imaging, vol. 12, no 2, suppl. 1, pp. 116-118, maio, 1999.

\section{[CLUTCHER-1990]}

CLUTCHER, B.B.; SCHERPBIER H. Benefits of an integrated hospital information system. American Journal of Hospital Pharmacy, vol. 47, pp. 779-780, 1990.

[COOK-1999]

COOK, J.F.; ROZENBLIT J.W.; CHACKO A.K.; MARTINEZ R.; TIMBOE H. L. Metamanager: A requirement analysis. Journal of Digital Imaging, vol. 12, $\mathrm{n}^{\mathrm{o}}$ 2, suppl. 1, pp. 186-188, maio, 1999.

[ELMASRI-1989]

ELMASRI, R; NAVATHE, S. Fundamentals Of Database Systems. The Benjamin Cummings Publishing Company, 1989.

[FERNÀNDEZ-BAYÓ-2000]

FERNÀNDEZ-BAYÓ, J.; BARBERO, O.; RUBIES C.; MELCIOR, S.; DONOSO, L. Distributing Medical Imagens with hternet Technologies: A DICOM Web Server and a DICOM Java Viewer. Radiographics, vol. 20, pp. 581-590, 2000.

[FOORD-1999]

FOORD, K.; TOMLINSON, N. The Conquest Hospital Picture Archiving and Communications System Development. Journal of Digital Imaging, vol. 12, nº 2, suppl. 
1, pp. 211-213, maio, 1999.

[FREIRE-1997]

FREIRE, H.J.P. Proposta de um sistema de banco de dados para tomografia por ressonância magnética nuclear. Dissertação de mestrado ao Instituto de Física de São Carlos - Universidade de São Paulo (USP), 1997.

[FRITZ-1999]

FRITZ, S.L. DICOM Standardization. Filmless Radiology, Springer Verlag, pp. 311-321, 1999.

[FURTADO-1987]

FURTADO, A.L.; SANTOS C.S. Organização de banco de dados. Campus, 1987.

[FURUIE-1999]

FURUIE, S.S.; BERTOZZO, N.B.; FIGUEIREDO, J.C.B.; YAMAGUTI M. Archiving and Retrieving Long-Term Cineangiographic Images in a PACS. Computer in Cardiology, pp. 435-438, 1999.

[GARLAND-1999]

GARLAND, H.T.; CAVANAUGH B.J.; CECIL R.; HAYES B.L.; LAVOIE S.; LEONTIEV A.; VEPRAUSKAS J. Interfacing the Radiology Information System to the Modality: An Integrated Approach. Journal of Digital Imaging, vol. 12, $\mathrm{n}^{\mathrm{o}}$ 2, suppl. 1, pp. 91-92, maio, 1999. 
[GARDNER-1999]

GARDNER, R.M.; PRYOR A.; WARNER H.R. The HELP hospital information system: update 1998. International Journal of Medical Informatics, vol. 54, pp. 169-182, 1999.

[HONDA-2001]

HONDA, M.O. Elaboração de uma base de conhecimentos para auxílio ao diagnóstico através da comparação visual de imagens mamográficas. Dissertação de mestrado a Escola de Engenharia de São Carlos - Departamento de Engenharia Elétrica - Universidade de São Paulo (USP), 2001.

[HONEYMAN-1999]

HONEYMAN, J.C. Information Systems Integration in Radiology. Journal of Digital Imaging, vol. 12, n 2, suppl. 1, pp. 218-222, maio, 1999.

[HWANG-2000]

HWANG, S.; LEE, M. A WEB-based TelePACS Using an Asymmetric Satellite System. IEEE Transactions on Information Technology in Biomedicine, vol. 4, $\mathrm{n}^{\mathrm{o}} 3$, pp. 212-215, setembro, 2000.

[JOHANSTON-1993]

JOHANSTON, H. Sistemas de Informação Hospitalar: Presente e Futuro. Revista Informédica, vol. 1, nº 2, pp. 5-9, 1993. 
[JOHNSON-2000]

JOHNSON, N.D.; GAROFOLO G.; GEERS W. Demystifying the Hospital Information System/Radiology Information System Integration Process. Journal of Digital Imaging, vol. 13, n 2, suppl. 1, pp. 175-179, maio, 2000.

[JUNCK-1998]

JUNCK, K.L.; BERLAND, L.L.; BERNREUTER, W.K.; MCEACHERN, M.; GRANDHI, S.; LEWEY, G. PACS and CR Implementation in a Level I Trauma Center Emergency Department. Journal of Digital Imaging, vol. 11, no 3, suppl. 1, pp. 159-162, agosto, 1998.

[KIMURA-1998]

KIMURA, M.; TANI, S.; BAATAR S.; NAITO Y.; KANNO, T.; SAKUSABE T.; AIZAWA, M. Implementation of multi-vendor DICOM standard image transfer in hospital wide ATM network. Computer Methods and Programs in Biomedicine, vol. 57, pp. 85-89, 1998.

[KORTH-1994]

KORTH, H.F.; SILBERSCHATZ, A. Sistema de Banco de Dados. Makron Books, 1994.

[KUZMAK-1998]

KUZMAK, P.M.; DAYHOFF, R.E. Integration of Imaging Functionality into the

Healthcare Enterprise using DICOM. Journal of Digital Imaging, vol. 11, no 3, suppl. 1, pp. 67-70, agosto, 1998. 
[NEMA-1999]

http://medical.nema.org/dicom.html. Acesso em 18/10/2000.

[OBERSON-2000]

OBERSON, J-C; WELZ, R.; BOVISI L. Development of an Electronic Radiologist's Office in a Private Institute. Inforad, vol. 20, pp. 573-580, março-abril 2000.

[OSADA-1999]

OSADA, M.; NISHIHARA E. Implementation and Evaluation of Workflow Based on Hospital Information System/Radiology Information System/Picture Archiving and Communications System. Journal of Digital Imaging, vol. 12, $\mathrm{n}^{\mathrm{o}}$ 2, suppl. 1, pp. 103-105, maio, 1999.

[PEER-1999]

PEER, S.; VOGL, R; PEER R.; JASCHKE, W. Sophisticated Hospital Information System/Radiology Information System/Picture Archiving and Communications System (PACS) Integration in a Large-Scale Traumatology PACS. Journal of Digital Imaging, vol. 12, nº 2, suppl. 1, pp. 99-102, maio, 1999.

[REDFERN-2000]

REDFERN, R.O.; HORII, S.C.; FEINGOLD E.; KUNDEL, H.L. Radiology Workflow and Patient Volume: Effect of Picture Archiving and Communications Systems on Technologists and Radiologists. Journal of Digital Imaging, vol. 13, $\mathrm{n}^{\mathrm{o}}$ 2, suppl. 1, pp. 97100, maio, 2000. 
[REINER-1998]

REINER, B.; SIEGEL, E.; HOOPER, F.; GLASSER, D. Effect of film-based versus filmless operation on the productivity of CT technologists. Radiology, vol. 207, pp. 481485, 1998.

[REINER-1999]

REINER，B.; SIEGEL， E.; PROTOPAPAS，Z.; HOOPER， F.; GHEBREKIDAN， H.; SCANLON, M. Impact of Filmless Radiology on Frequency of Clinician Consultations with Radiologists. American Roentgen Ray Society, vol. 173, pp. 1169-1172, novembro, 1999.

[REINER-2000]

REINER, B. I.; SIEGEL E. L.; FLAGLE, C.; HOOPER, F. J.; COX, R. E.; SCANLON, M. Effect of filmless imaging on the utilization of radiologic services. Radiology, vol. 215, pp. 163-167, 2000.

[RSNA-1997]

www.rsna.org/REG/practiceres/dicom/nontechintro.html. Acesso em 10/10/2000.

\section{[SHENG-1990]}

SHENG, O. R. L.; et al. Requirement Analysis for PACS database system. SPIE Medical Imaging IV: PACS System Design and Evaluation, vol. 1234, pp. 842-854, 1990. 
[SIEGEL-1998]

SIEGEL, E. L. Economic and Clinical Impact of Filmless Operation in a Multifacility

Environment. Journal of Digital Imaging, vol. 11, $\mathrm{n}^{\mathrm{o}}$ 4, suppl. 2, pp. 42-47, novembro, 1998.

[SIEGEL-1999]

SIEGEL E. L.; KOLODNER, R. M. Filmless Radiology: State of the Arte and Future Trends - in Filmless Radiology. Siegel and Kolodner eds - Springer-Verlag, pp.3-20, 177 e 208, 1999.

[STEVE-2000]

STEVE, G. L. Architecture of an Image Capacle, Web-Based, Electronic Medical Record. Journal of Digital Imaging, vol. 13, nº 2, pp. 82-89, maio, 2000.

[STRICKLAND-1999]

STRICKLAND, N. H. Can PACS make a radiology department more competitive?. European Journal of Radiology, vol. 32, pp. 113-115, agosto, 1999.

[TAMM-2000]

TAMM, E.P.; ZELITT D.; DINWIDDIE, S. Implementation and Day-to-Day Usage of a Client-Server-Based Radiology Information System. Journal of Digital Imaging, vol. 13, $\mathrm{n}^{\mathrm{o}}$ 2, suppl. 1, pp. 213-214, maio, 2000. 
[WANG-2000]

WANG, S.S.; STARREN, J. A Web-Based, Secure, Light Weight Clinical Multimedia

Data Capture and Display System. Departments of Medical Informatics and Radiology, Columbia University, New York. 